\title{
Optimization techniques in respiratory control system models
}

\author{
Leidy Y. Serna ${ }^{1}$, Miguel Ángel Mañanas ${ }^{1}$, Jesús Marín ${ }^{1}$, Alher Mauricio Hernández ${ }^{2}$, \\ Salvador Benito ${ }^{3}$
}

\begin{abstract}
One of the most complex physiological systems whose modeling is still an open study is the respiratory control system where different models have been proposed based on the criterion of minimizing the work of breathing (WOB). The aim of this study is twofold: to compare two known models of the respiratory control system which set the breathing pattern based on quantifying the respiratory work; and to assess the influence of using direct-search or evolutionary optimization algorithms on adjustment of model parameters. This study was carried out using experimental data from a group of healthy volunteers under $\mathrm{CO}_{2}$ incremental inhalation, which were used to adjust the model parameters and to evaluate how much the equations of WOB follow a real breathing pattern. This breathing pattern was characterized by the following variables: tidal volume, inspiratory and expiratory time duration and total minute ventilation. Different optimization algorithms were considered to determine the most appropriate model from physiological viewpoint. Algorithms were used for a double optimization: firstly, to minimize the WOB and secondly to adjust model parameters. The performance of optimization algorithms was also evaluated in terms of convergence rate, solution accuracy and precision. Results showed strong differences in the performance of opti-
\end{abstract}

\footnotetext{
${ }^{1}$ L. Y. Serna, M.A. Mañanas and J. Marín are with Dept. Automatic Control, ESAII, Biomed. Eng. Research Center, CREB and the Biomedical Research Networking Center in Bioengineering, Biomaterials and Nanomedicine, CIBER-BBN,. Technical University of Catalonia, Barcelona, Spain (leidy.yanet.serna@upc.edu, miguel.angel.mananas@upc.edu and jesus.marin-sanchez@upc.edu, respectively)

${ }^{2}$ A. M. Hernández is with the Bioengineering Program, Bioinstrumentation and Clinical Eng. Group, University of Antioquia, Medellín, Colombia (mauricio.hernandez@udea.edu.co).

${ }^{3}$ S. Benito is with the Santa Creu i Sant Pau Hospital of Barcelona and the Department of Medicine, Universitat Autònoma de Barcelona, Spain (SBenito@santpau.cat)
} 
mization algorithms according to constraints and topological features of the function to be optimized. In breathing pattern optimization, the sequential quadratic programming technique (SQP) showed the best performance and convergence speed when respiratory work was low. In addition, SQP allowed to implement multiple non-linear constraints through mathematical expressions in the easiest way. Regarding parameter adjustment of the model to experimental data, the evolutionary strategy with covariance matrix and adaptation (CMA-ES) provided the best quality solutions with fast convergence and the best accuracy and precision in both models. CMAES reached the best adjustment because of its good performance on noise and multi-peaked fitness functions. Although one of the studied models has been much more commonly used to simulate respiratory response to $\mathrm{CO}_{2}$ inhalation, results showed that an alternative model has a more appropriate cost function to minimize WOB from a physiological viewpoint according to experimental data.

Keywords: Respiratory control system, optimal control, optimization algorithms, mechanical work of breathing

\section{Introduction}

The primary function of respiratory system is to regulate the homeostasis of arterial blood gases and $\mathrm{pH}$, through supplying oxygen to the blood and removing carbon dioxide $\left(\mathrm{CO}_{2}\right)$ produced by metabolic activities. From a modeling viewpoint, the respiratory system can be considered as a neurodynamic feedback system, nonlinear, multivariable with delays and continuously affected by physiological and pathological disturbances. Its behavior can be defined by a continuous interaction between the controller and peripheral processes are being controlled (respiratory mechanics and pulmonary gas exchange). The peripheral processes have been extensively studied in previous research [1, 2, 3, 4, 5, 6, 7]. Nevertheless, respiratory controller behavior and how the controller processes different afferent inputs are not completely inferred yet [8].

One of the most interesting issues concerning respiratory system modeling is the possibility of forecasting the respiratory system response of a critical patient connected 
to a mechanical ventilator. However, mathematical models are still far from allowing this, mainly because of the complexity of the respiratory control system that adjusts the breathing pattern according to mechanical and chemical components minimizing the work of breathing (WOB) and the system response can be affected either by the cost function or by the optimization technique.

The aim of this study is twofold: firstly, to compare two known mechanical cost functions to quantify the WOB [9] and, secondly, to assess the influence of several known optimization techniques, such as direct search and evolutionary algorithms $[10$, $11,12,13,14,15,16,17,18,19$, on the adjustment of the breathing pattern (by the controller) and the model parameters (in the identification process).

Two nested optimizations were carried out for this purpose: the first one in the controller that minimizes the WOB by using both mechanical cost estimations proposed in [9] and the second one in the search of the model parameters associated with the response that better matched with experimental data. These data were recorded from a group of subjects under different levels of ventilation $\left(\dot{V}_{E}\right)$ produced by hypercapnic stimulation. Hypercapnia is characterized by changes in partial pressure of $\mathrm{CO}_{2}$ in arterial blood $\left(\mathrm{PaCO}_{2}\right)$ above the normal value $(40 \mathrm{mmHg})$, so the neural control center respond adjusting $\dot{V}_{E}$ and, therefore, breathing pattern subject, in order to keep $P_{a C O}$ near physiological values. In this study, hypercapnia was produced increasing partial inspiratory pressure of $\mathrm{CO}_{2}\left(\mathrm{P}_{\mathrm{ICO}_{2}}\right)$.

Known evolutionary and deterministic optimization algorithms are applied in order to identify the best option for this kind of optimization problems and to guarantee that the model parameter adjustment allows to reproduce real situations with physiological meaning. The performance of these optimization algorithms are evaluated regarding the goodness of fit to experimental data, the convergence rate and the dispersion of the found solutions.

The paper is organized as follows. Section 2 presents previous studies about modeling of respiratory control system response and concerning optimization procedures used in biomedical applications. Section 3 presents a general description of the algorithms addressed in this study and their selected parameter values. Section 4 presents a mathematical description of the analyzed model and its variables of interest. After- 
wards, Section 5 shows the optimization problem to be solved: experimental data for the model adjustment, the two nested optimizations solved in this approach (the breathing pattern fitting and the model fitting to experimental data), and finally, statistical tests and validation procedure to select the best algorithms and to compare the found solutions. Section 6 presents the results associated to the both fittings. Finally, section 7 discusses and concludes the results found in the optimization problem and provides their interpretation in the respiratory model from a physiological point of view. A step by step description about how this approach was carried out is presented in the Fig. 1 .

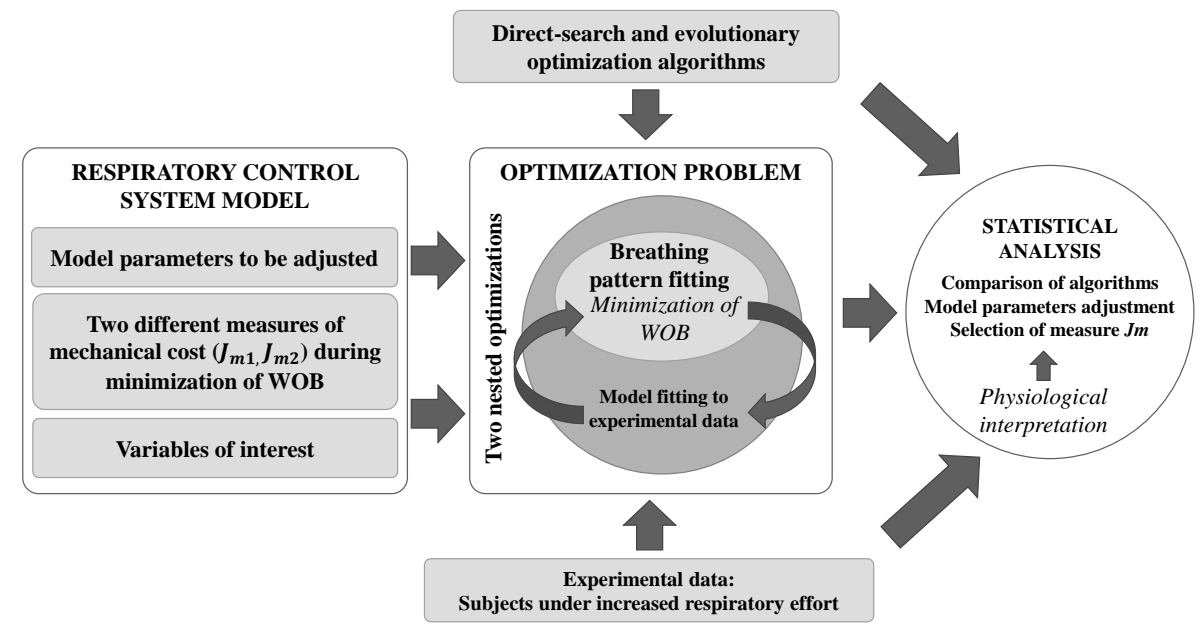

Figure 1: Description step by step how the approach is realized

\section{Related work}

\subsection{Respiratory control system modeling}

The respiratory controller may be seen as a central pattern generator in which rhythmic respiratory activity is produced in response to different afferent pathways [20]. Following this hypothesis, several approaches have been used to simulate this control law in respiratory control modeling. In this sense, some authors consider respiratory control system as a reflex-mechanism where breathing pattern is adjusted, from mathematical relationships obtained empirically, in order to meet a ventilatory demand [1, 21, 22]. This adjustment is carried out varying either respiratory frequency or volume or both. 
The minimization of WOB has been extensively considered as a control criterion to adjust the breathing pattern [23, 24, 25, 26, 27, 28, 29]. Using this approach, several models have been proposed using minute ventilation as controlled variable. Early formulations were based on sinusoidal airflow patterns in which respiratory frequency was fitted in function of minimum work rate criteria [30, 23] and minimum average driving pressure [31, 32]. Optimal criteria were also developed to predict airflow patterns. One of these criteria used the integral of the square of volume acceleration as cost function for both breathing phases with constant inspiration and expiration intervals [33].

Models of overall control of the breathing pattern using a two-level optimization problem have been also presented in [34, 35, 9].Particularly, Poon et al. [9] proposed a mathematical model of respiratory control system based on minimization of WOB which includes mechanical and chemical costs of breathing and allows to optimize simultaneously ventilation and breathing pattern (detailed information is presented in Section 4). This model has been used in studies related to respiratory modeling not only by the same author [28, 36, 37] but also by others [38, 39, 40]. Recently, several studies have used its cost functions to determine the breathing pattern in subjects under assisted mechanical ventilation [39, 41, 42, 43]. The WOB depended on model parameters whose values were not provided in any of the previous studies $[9,28,36,37,38,39,40,41,42,43]$, which suggests that respiratory control modeling based on optimization criteria needs additional experimental and computational efforts. In addition, two different functions were also proposed in [9] to measure the mechanical cost. Thus, our purpose is to analyze this model to find out the best of the two proposed mechanical functions and to adjust the best values of the model parameters according to experimental data. This analysis required two coupling and nested minimizations: WOB and differences between simulated and experimental respiratory responses. Finally, both fittings also required optimization algorithms which can affect parameter estimation and, with that, the WOB computation, thus, different approaches were compared based on the found solutions.

In addition, deep knowledge about the breathing pattern during increased ventilatory needs and its relationship with WOB is an interesting issue in mechanical ventilation and therapy of respiratory disorders [44, 45, 46]. In these situations, forecasting 
tools based on computationally-driven systems and suitable approaches to quantify WOB could be applied in order to improve patient treatments [47, 48]

\subsection{Optimization techniques in biomedical applications}

Optimization procedures have attracted a great deal of attention from research community, since many problems arising from diverse fields of knowledge can be posed and solved through mathematical programming techniques. Initially, local optimization methods were developed based on gradient techniques under assumption of convexity for solving nonlinear problems. Sequential Quadratic Programming (SQP) method is the most successful local optimization method for solving nonlinearly constrained optimization problems [49]. This method is frequently used in issues related to biomedical field, however, its use is restricted to convex problems so it is less efficient in problems that exhibit multiple local optima (most of them). For overcoming this handicap, global optimization algorithms, using determinist and stochastic approaches, were developed later. During the last decades, the interest in the use of such optimization techniques has been increasing, encouraging further analysis of their performance in solving complex problems in biomedical applications.

In the past years, the use of stochastic algorithms for global optimization such as Genetic Algorithm (GA), Particle Swarm Optimization (PSO), Differential Evolution (DE), Real-coded CHC, Covariance Matrix Adaptation Evolution Strategy (CMA-ES) among others have been applied in a large variety of studies concerning biomedical field. For example:

- Lambrou et al. [50] developed a conformal prediction (CP) based on GAs and applied their method on two medical diagnosis problem. The study results showed the efficiency of their method on both problems in term of accuracy. Ghosh et al. [51] implemented a nonlinear autoregressive model of blood glucose metabolism using GA to represent the dynamics of glucose utilization following an intravenous glucose tolerance test. In such study, the model did not need an initial parameterization and the convergence was always guaranteed.

- Selvan et al. [52] presented a new approach based on PSO and evolutionary pro- 
gramming (EP) for accurately estimating the parameter set of a stochastic mammogram model. The experimental results show important advantages regarding to the conventional algorithm.

- Ghosh [53] using DE algorithm for the determination of insulin sensitivity form the minimal model using clinical test data. The estimation process was formulated as an optimization problem by minimizing error between experimental and model output data.

- Cordón et al. [54] introduced the use of CHC to face the feature-based 3D image registration problem and concluded that $\mathrm{CHC}$ solutions outperform those obtained by the other approaches when considering both noisy and noise-free scenarios. On other hand, Lee and Nelson [55] used the CHC algorithm to found optimal feature weights of a pattern recognition technique for the prediction of contrast-enhancement appearance in brain tumors from multivariate magnetic resonance imaging and spectroscopy.

- Moscardó et al [56] used the global optimization algorithm CMA-ES for estimation of parameters of an adrenaline secretion model during hypoglycemia prediction in type 1 diabetes, whereas Aler et al. [57] used the same algorithm for preprocessing electroencephalogram (EEG) signals for brain-computer interface.

Additionally, determinist methods for global optimization, like Generalized Pattern Search (GPS) and Mesh Adaptive Direct Search (MADS), are generally used with stochastic optimization algorithms. Combinations of both of them are usually implemented. For instance, Das et al. [58] used MADS and a genetic algorithm (GA) to compute optimal setting of mechanical ventilator parameters from minimizing two objective functions. On the other hand, Zhang et al. [59] implemented an hybrid optimization algorithm based on GPS and GA to brain image classification. The hybrid model improved the robustness of GPS and the convergence speed of GA. Yang et al. [60] used the surrogate management framework (SMF, a derivate-free optimization method) with MADS to the shape of a developed surgical design for the Fontan proce- 
dure.

Some studies where performance of several optimization techniques are evaluated regarding to robustness and convergence speed have been recently presented. Ros et al. [61] presented a comparison among stochastic optimization algorithms for parameter estimation of biochemical kinetic models. In such study, Artificial Bee Colony (ABC), DE, PSO and Simulated Annealing (SA) algorithms were compared. The results indicated that DE provide better solutions than the others analyzed algorithms. Ahirwal et al. [62] analyzed the effect of different optimization algorithms in the adaptive noise cancellation (ANC) for event related potential filtering from EEG signals: gradient methods like least mean square (LMS), normalized least mean square (NLMS), recursive least square (RLS) and evolutionary algorithms like PSO, GA among others. Simulation results showed the key advantageous features of the ANC based on PSO and other evolutionary technique over others in the field of biomedical signal processing. Zimmer and Sahle [63] compared generic least square (LSQ) and specialized (MSS) approaches for parameter estimation on stochastic models. In this study, MSS methods showed a lot better performance regarding to LSQ methods. The former was able to capture the most important features of analyzed model.

\section{Optimization algorithms}

Optimization techniques involve the selection of the best element, regarding some criteria, from a set of available alternatives. In this sense, optimization algorithms are tools which try to minimize (sometimes maximize) a cost function or error by systematically choosing input parameters within a search space. These input parameters involve variables which are often restricted or constrained. There is not a universal optimization algorithm, so the choice of an appropriate algorithm for a specific real problem is important to obtain feasible solutions [64].

Optimization algorithms can be classified according to different approaches. One is depending on the focus or the characteristics that are trying to compare. They can be classified as gradient-based (or derivative-based) methods and gradient-free (or derivative-free) methods or as local and global optimization methods, where the former 
typically converge towards a local minimum, which is not necessarily the global minimum, and they have no ability of escaping local optima. On the other hand, optimization algorithms can be also classified as deterministic or stochastic. A deterministic algorithm works with a mechanical deterministic procedure without any random nature, so it reaches always the same final solution if it starts with the same initial point. A stochastic algorithm has random behavior, so it usually reaches a different point every time the algorithm is run and it is generally a global optimization method [64].

Direct-search algorithms are a class of gradient-free and deterministic method. Unlike more traditional optimization methods that use information about the gradient search for an optimal point, a direct-search algorithm searches a set of points around the current point, looking for one point where the value of the objective function is lower than the value at the current point.

Evolutionary algorithms (EA) are stochastic optimization algorithms which include real-coding inspired by nature processes, such as reproduction, mutation, recombination and selection [65]. They work with a population of individuals or a set of candidate solutions, where each individual includes a genotype represented as a vector of realcoded inputs, and also a fitness value, which is obtained applying the cost function (or error) to these inputs. The population is iteratively updated (by reproduction) at each evaluation or generation by applying selection, crossover and/or mutation operators, which are controlled by algorithm parameters. Each method implements its own strategy to optimize avoiding a premature convergence to local optimal.

Evolution strategies (ES) are EAs based on ideas of adaptation and evolution; they are characterized by an elitist selection making that the fittest individuals are selected to reproduce [65]. ES are iterative (generational) procedures, where in each generation new individuals (offspring) are created from existing individuals (parents). The notations $(\mu / \rho, \lambda)-E S$ or $(\mu / \rho+\lambda)-E S$ are commonly used to describe some aspects of this interaction. In these expressions, $\mu, \rho$ and $\lambda$ are positive integers that represent the parent population, size of population used for recombination $(\rho \leq \mu)$ and the number of offspring generated in each iteration, respectively. Comma-selection denotes the parents are chosen from the multi-set of both parents and offspring (age is not taken into account), whereas in plus-selection the parents are chosen only from sets of 
offspring.

In this study, evolutionary and direct-search algorithms which had been successfully used in optimization problems in the biomedical field [50, 51, 52, 53, 54, 55, 56, 57, 58, 59, 60, 61, 62, 63] were selected for their comparison. Simulations were performed fixing as stopping criterion a maximum number of evaluations of the analyzed cost functions. Particularly, the performance of the following algorithms was compared using the settings suggested for their authors:

- Sequential Quadratic Programming (SQP) algorithm [11], a deterministic and gradient-based algorithm. It is an interactive method for nonlinear optimization and it is used on problems where the objective function and the constraints are both continuously differentiable.

- Generalized Pattern Search (GPS) algorithm, which is a deterministic and global optimization algorithm, has been defined by [12, 13] for gradient-free unconstrained and linearly constrained optimization on continuously differentiable functions using positive spanning directions. GPS searches a set of values around of an initial point using a finite set of fixed direction vectors, called pattern, and it selects the point with the lowest value. Then, the seudo-optimal point is used as the new initial point around which the new pattern will be constructed again. This process is iteratively repeated until one of the convergence criteria is accomplished. Due to its behavior GPS is also known as a direct-search method [66].

- Mesh Adaptive Search (MADS) algorithm [14] extends the GPS algorithm by allowing local exploration, called polling, in an asymptotically dense set of directions in the space of optimization variables. A key advantage of MADS over GPS, for both unconstrained and linearly constrained optimization, is that local exploration of the space of variables is not restricted to a finite number of directions. The searching process is like in GPS but using a random selections of vectors instead fixed direction vectors.

- Real-coded CHC algorithm [15] (Cross-generational elitist selection, Heterogeneous recombination, and Cataclysmic mutation), which is an EA with binary 
coding tries to avoid a premature convergence through a suitable balance between the ability to explore the search space for diversity and the ability to exploit the local properties of the search for an appropriate selection [67]. It combines a highly disruptive recombination operator with an elitist selection operator and an incest prevention mechanism for good converge velocity. In this study, $B L X-\alpha$ was used as crossover operator.

- Macroevolutionary Algorithm (MA-ES) algorithm [16], which is a variant of $(\mu / 2+\lambda)$, uses a model of a network ecosystem where the dynamics are based only on the relationship between species. This relationship is used to determine the new state (alive or extinct) of each species at each generation.

- Differential Evolution (DE) algorithm [17], which is an EA proposed to solve optimization problems mainly in continuous search spaces. This performs mutation based on the distribution of the solutions in the current population, searches directions and possible step sizes depending on the location of the individuals selected to calculate the mutation values [68]. In this study, the variant $\mathrm{DE} / \mathrm{rand} / 1 /$ bin was used.

- Particle Swarm Optimization (PSO) algorithm [18] is an EA that uses a method inspired by collective "swarming" and "flocking" of sociable animals. In this algorithm, the individuals are particles moving around in the search-space according to position and velocity. Each particle's movement is influenced by its local best position with respect to the pseudo-optimal and the best found position of all "swarming" and "flocking" particles. Some examples of practical applications can be found in [69].

- Evolution strategy with Covariance Matrix Adaptation (CMA-ES) algorithm [19], which is a $(\mu / \mu, \lambda)$-ES that employs a technique where the covariance matrix of the distribution is updated (incrementally) such that the likelihood of previously successful search steps is increased. In consequence, the CMA-ES conducts an iterative principal components analysis of successful search steps instead of successful solution points. In this work, CMA-ES is used in conjunction with 
the increasing population-size restart policy (IPOP-CMA-ES) proposed by [70], which has shown good performance on multi-peaked functions and noisy testbed and with $\mu=\lambda / 2$ where $\lambda$ is the population size.

All these algorithms were run in MATLAB version 7.12. SQP, GPS and MADS using the global optimization toolbox; DE, PSO and CMA-ES using the source codes available at [71, 72, 73], respectively; and CHC and MA-ES using the source codes available at [74].

These algorithms were applied using the parameter values suggested by each author and some of them were modified in order to improve their performance. Tab. 1 shows the values used in this study. More information of these parameters can be found in the source code and previous references. With the aim of guarantee a reasonable comparison, the stop criterion for all algorithms was the maximum number of functions evaluations which was set to 400 . This value permitted the convergence of the most algorithms as it will be shown in the results to compare their performance.

Due to deterministic algorithms, such as SQP, GPS and MADS, are potentially sensitive to the initial conditions to start the search, the initial solutions were randomlygenerated with uniform distributions and the seed used by a pseudo-random generator was fixed with the same value for all algorithms to be compared with similar conditions. Moreover, when a premature convergence to a minimal local was presented an automatic random re-initialization was applied to improve the reached solution, if it was possible. Finally, in order to guarantee the reproducibility of results, the optimization processes exposed in this study were run 25 times for each case 


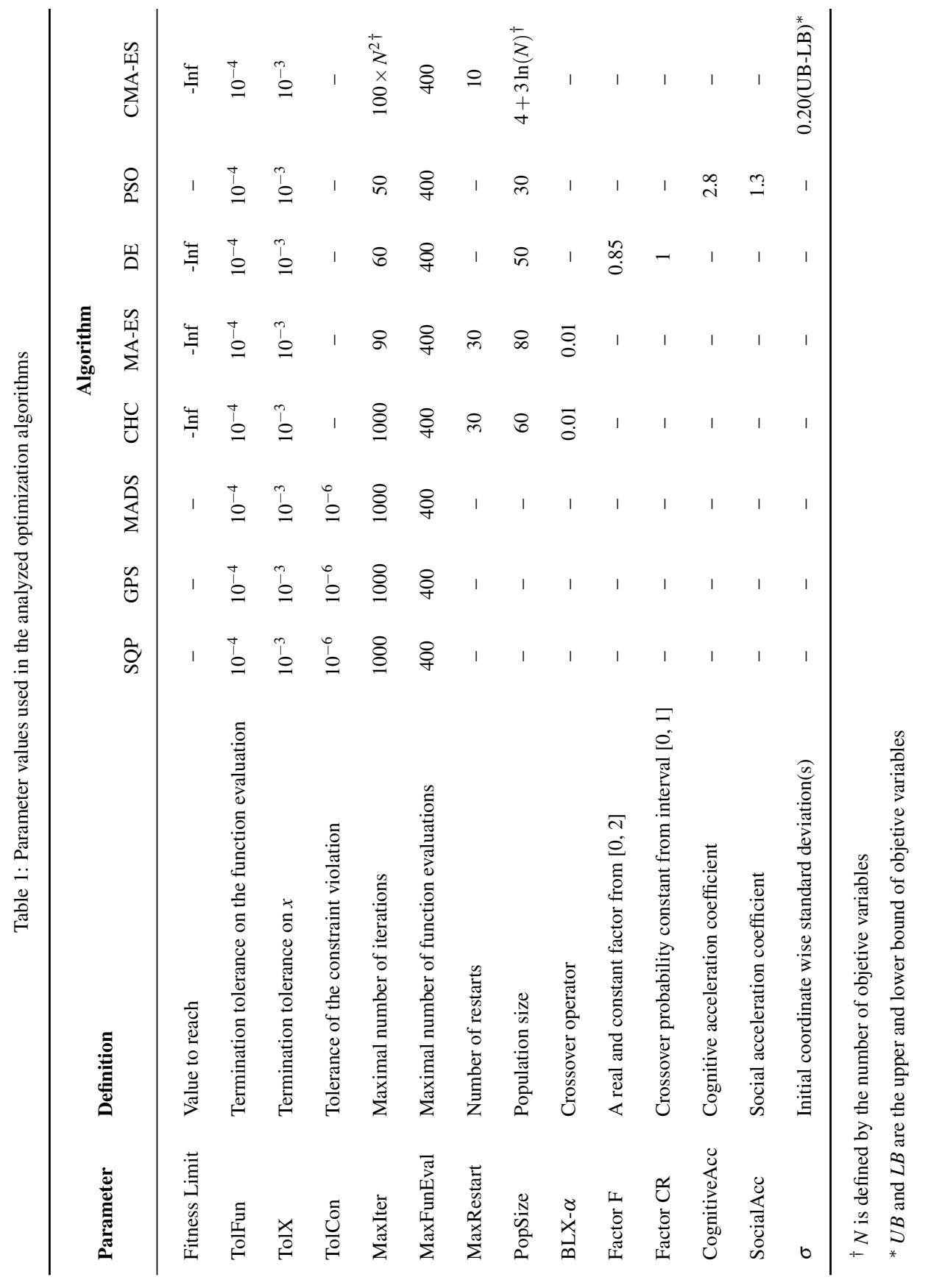




\section{Respiratory control system model}

\subsection{Model description}

The model used in this work was proposed by [9] to describe the stationary response of respiratory system under hypercapnia and exercise stimuli. This model presents an optimal control that adjusts $\dot{V}_{E}$ and breathing pattern as a function of minimization of WOB and includes dynamic elements that relate neural activity to ventilatory mechanics [75]. The model discriminates between the mechanical work carried out during inspiration and expiration, so that not only adjusts $\dot{V}_{E}$ but also the set of variables associated with the breathing pattern in terms of the minimum WOB. It also takes into account important variables such as respiratory frequency $\left(f_{R}\right)$, tidal volume $\left(V_{T}\right)$ and inspiratory and expiratory time intervals ( $T_{I}$ and $T_{E}$ respectively).

The respiratory system is modeled as a closed-loop feedback control system comprising five major subsystems: the controlled system (chemical plant, i.e. gas exchanger), the chemical feedback path (chemoreceptors), the mechanical feedback path (work rate index), the controller (medulla oblongata) and the actuator/effector (neuromechanical effector) as it is shown in Fig. 2 .

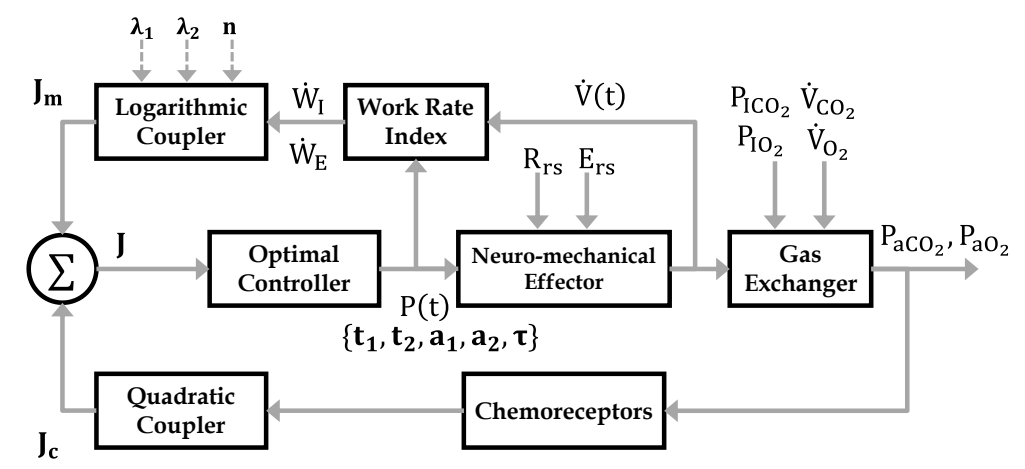

Figure 2: Block diagram of the model of the respiratory control system proposed by [9].

As it can be seen in Fig. 22, the respiratory cost function $(J)$ in this model has two components:

$$
J=J_{c}+J_{m}=\alpha^{2}\left(P_{a C O_{2}}-\beta\right)^{2}+\ln \left(\dot{W}_{I}+\lambda_{2} \dot{W}_{E}\right)
$$


The first component is the chemical cost $\left(J_{c}\right)$ where $\alpha$ is the chemoreceptor sensitivity and $\beta$ the chemoreceptor response threshold. From mass balance equation, which describes the exchange of gases of $\mathrm{CO}_{2}$ and $\mathrm{O}_{2}, \mathrm{PaCO}_{2}$ is obtained as following:

$$
P_{a C O_{2}}=P_{I C O_{2}}-\frac{863 \dot{V}_{C O_{2}}}{\dot{V}_{E}\left(1-V_{D} / V_{T}\right)}
$$

where $\dot{V}_{\mathrm{CO}_{2}}$ is the metabolic rate of $\mathrm{CO}_{2}$ and $V_{D}$ the dead space volume. $V_{D}$ is calculated from vital capacity, $V C=V_{T}+4$ in liters, and $V_{T}$ as follows:

$$
V_{D}=0.037 \times V C\left(1+V_{T} / 8\right)
$$

In this model, $\mathrm{PaCO}_{2}$ is assumed to be identical to mean alveolar pressure of $\mathrm{CO}_{2}$, so Eq. 2 describes the steady state effect of ventilation on $\mathrm{PaCO}_{2}$.

The second term in Eq. 1 represents the mechanical cost $\left(J_{m}\right)$. This cost discriminates between the work performed during inspiration $\left(\dot{W}_{I}\right)$ and expiration $\left(\dot{W}_{E}\right)$ and weights their contributions by using the parameter $\lambda_{2}$. $\dot{W}_{I}$ and $\dot{W}_{E}$ are determined by the following set of equations [9]:

$$
\begin{gathered}
\dot{W}_{I}=\frac{1}{T_{T O T}} \int_{0}^{T_{I}} \frac{P(t) \dot{V}(t)}{\xi_{1}^{n} \xi_{2}^{n}} d t \\
\dot{W}_{E}=\frac{1}{T_{T O T}} \int_{T_{I}}^{T_{T O T}} P(t) \dot{V}(t) d t \\
\dot{W}_{I}=\frac{1}{T_{T O T}} \int_{0}^{T_{I}}\left[\frac{P(t)}{\xi_{1}^{n} \xi_{2}^{n}}+\lambda_{1} \ddot{V}(t)^{2}\right] d t \\
\dot{W}_{E}=\frac{1}{T_{T O T}} \int_{T_{I}}^{T_{T O T}} \ddot{V}(t)^{2} d t
\end{gathered}
$$

where $P(t), V(t), \dot{V}(t)$ and $\ddot{V}(t)$ denote respiratory muscle pressure, volume, airflow and volume acceleration respectively and $\lambda_{1}$ is a weighting factor. $P(t)$ and $V(t)$ 
are measured at functional residual capacity (FRC).In Eqs. 4a and 5a $n$ represents nonlinear variation of efficiency and $\xi_{1}$ and $\xi_{2}$ are mechanical efficiency factors defined as:

$$
\xi_{1}=1-\frac{P(t)}{P_{\max }} \quad \xi_{2}=1-\frac{\dot{P}(t)}{\dot{P}_{\max }}
$$

Eqs. 4 and 5 describe two approaches to quantify WOB. In this paper RSM1 denotes the respiratory system model comprising by Eqs. $4 \mathrm{a}$ and $4 \mathrm{~b}$ for inspiratory and expiratory work, respectively; and RSM2 is analogously defined by Eqs. $5 \mathrm{a}$ and $5 \mathrm{~b}$. Although Eqs. 5a and 5b do not measure exactly WOB, their minimization implies decreasing $P(t)$ and $\ddot{V}(t)$ associated with a minimum respiratory effort according to [24, 9].

In [9], $P(t)$ is defined as a piecewise function: a quadratic function during inspiration and exponential function during expiration. Formally,

$$
\begin{gathered}
P(t)=a_{0}+a_{1} t+a_{2} t^{2} \quad \text { for } \quad 0 \leq t \leq t_{1} \\
P(t)=P\left(t_{1}\right) e^{-\left(t-t_{1}\right) / \tau} \quad \text { for } \quad t_{1}<t \leq t_{1}+t_{2}
\end{gathered}
$$

where $t_{1}$ and $t_{2}$ are neural inspiratory and expiratory duration; $a_{0}, a_{1}$ and $a_{2}$ represent, respectively, the net drive pressure, its rate at the onset of neural inspiratory phase and the shape of the pressure wave (concave when $a_{2}<0$ and convex upward when $\left.a_{2}>0\right)$.

$P(t), V(t)$ and $\dot{V}(t)$ are related by the air movement equation, as follows:

$$
P(t)=R_{r s} \dot{V}(t)+E_{r s} V(t)
$$

where $R_{r s}$ and $E_{r s}$ correspond to the total flow-resistance and elastance components of the respiratory system respectively. From Eq. 8 volume can be obtained substituting $P(t)$ and solving for $V(t)$, following equations show resulting for inspiration and expiration phases:

$$
\begin{aligned}
V(t)= & {\left[A_{1} t+A_{2} t^{2}+A_{3}\left(1-e^{\frac{-t}{\tau_{r s}}}\right)\right] \frac{\tau_{r s}}{R_{r s}} } \\
& +V_{0} e^{\frac{-t}{\tau_{r s}}} \text { for } 0 \leq t \leq t_{1}
\end{aligned}
$$




$$
\begin{aligned}
V(t)= & \frac{P\left(t_{1}\right)}{R_{r} s\left(\frac{1}{\tau_{r s}}-\frac{1}{\tau}\right)}\left[e^{\frac{t_{1}-t}{\tau}}-e^{\frac{t_{1}-t}{\tau_{r s}}}\right] \\
& +V\left(t_{1}\right) e^{\frac{t_{1}-t}{\tau_{r s}}} \text { for } t_{1}<t \leq t_{1}+t_{2}
\end{aligned}
$$

where $V_{0}$ is the volume at the beginning of inspiration and $\tau_{r s}$ is the time constant of respiratory mechanical system given by $R_{r s} / E_{r s}$. The simplified terms of these expressions, considering the $a_{0}$ term in Eq.7a equals zero, are:

$$
A_{1}=a_{1}-2 a_{2} \tau_{r s} \quad A_{2}=a_{2} \quad A_{3}=a_{1} \tau_{r s}-2 a_{2} \tau_{r s}^{2}
$$

Fig. 3 shows pressure, volume and airflow waveforms for three levels of $P_{I_{C O}}$. It can be seen that $P(t), V(t), \dot{V}(t)$ and $f_{R}$ increase with level of stimulus, whereas $T_{I}$ decreases.

(a)

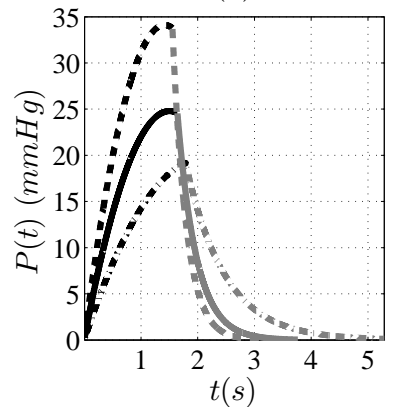

(b)

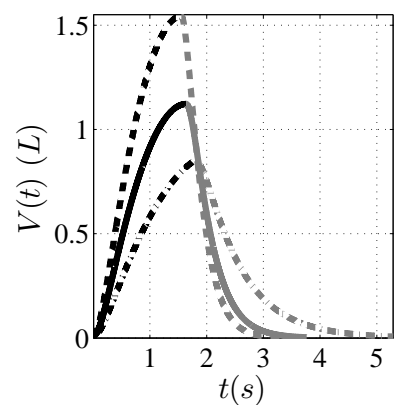

(c)

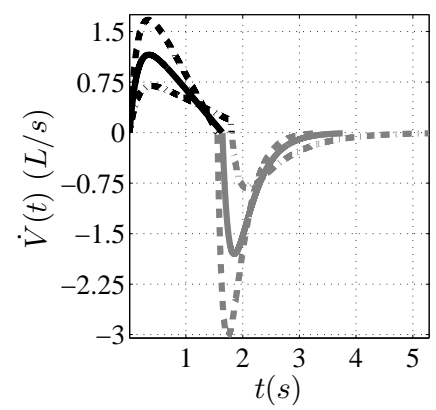

Figure 3: Pressure $P(t)$ (a), volume $V(t)$ (b) and airflow $\dot{V}(t)$ (c) waveforms during a ventilatory cycle for different levels of stimuli according to [9]. Black traces correspond to inspiratory phase and gray traces to expiratory phase

In order to simulate the model, the values of $\alpha, \beta, R_{r s}, E_{r s}$ and $\dot{P}_{\max }$ were taken from [9] for healthy subjects. $P_{\max }$ was set to $50 \mathrm{cmH}_{2} \mathrm{O}$ because higher pressures can increase barotrauma risk both in healthy and pathological subjects [76, 77]. Tab. 2 shows the value considered for each parameter. 


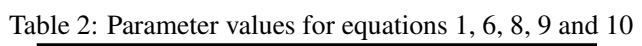

\begin{tabular}{lll} 
Parameter & Value & Units \\
\hline$\alpha$ & 0.0934 & - \\
$\beta$ & 37.78 & $\mathrm{mmHg}$ \\
$R_{r s}$ & 3.02 & $\mathrm{cmH}_{2} \mathrm{O} \cdot 1^{-1} \mathrm{~s}$ \\
$E_{r s}$ & 21.9 & $\mathrm{cmH}_{2} \mathrm{O} \cdot 1^{-1}$ \\
$\dot{P}_{\max }$ & 1000 & $\mathrm{cmH}_{2} \mathrm{O} / \mathrm{s}$ \\
$P_{\max }$ & 50 & $\mathrm{cmH}_{2} \mathrm{O}$ \\
\hline
\end{tabular}

\subsection{Variables of interest}

The main function of respiratory system is to adjust $\dot{V}_{E}$ to keep $P_{a C O_{2}}$ and $P_{a O_{2}}$ levels within physiological values [78, 26, 27], so $\dot{V}_{E}$ was selected as independent variable. This variable, expressed in $\mathrm{L} / \mathrm{min}$, was used to analyze the stationary responses of model under study and it was calculated from product of $f_{R}$ in breath/min and $V_{T}$ in $\mathrm{L}$, as follows:

$$
\dot{V}_{E}=V_{T} \times f_{R}=\frac{V_{T}}{T_{T O T}} \times 60=\frac{V_{T}}{T_{I}+T_{E}} \times 60
$$

Where $T_{T O T}$, which is the inverse of $f_{R}$, is the respiratory cycle duration in seconds (s) and can also be obtained from the sum of $T_{I}$ and $T_{E}$. Relationship among $\dot{V}_{E}, V_{T}$, $f_{R}, T_{T O T}, T_{I}$ and $T_{E}$ means that only three of them are enough to represent the temporal characteristics of the airflow, so that $V_{T}$ and $T_{I}$ were chosen as dependent variables.

Using $T_{I}$ and $V_{T}$ as dependent variables and $\dot{V}_{E}$ as independent variable allows quantifying the ventilatory strategy used by the respiratory control system to adjust the breathing pattern and to regulate the blood gases. This strategy could be carried out increasing $f_{R}$ (which is related to $T_{I}$ ) or $V_{T}$ or both.

\section{Optimization problem}

The model response to ventilatory stimuli has to guarantee both the minimum respiratory $\operatorname{cost}(J)$ (Eq. 1) and a good fit to the study population. Therefore, two nested opti- 
mization processes were involved in the analyzed model: the optimization of breathing pattern by minimizing $J$ in function of $\left[t_{1}, t_{2}, a_{1}, a_{2}, \tau\right]$ and the fitting model response to experimental data by minimizing differences between experimental and simulated variables by fitting the parameters $\left[\lambda_{1}, \lambda_{2}, n\right]$. A description of experimental data and protocol as well as an explanation about how the two nested optimization processes were addressed in this study are given in the following subsections.

\subsection{Experimental data and protocol}

A database of eight male healthy volunteers [age (yr.): 31,9 $\pm 7,7$; height $(\mathrm{cm})$ : $175,7 \pm 4,3$; weight $(\mathrm{kg}): 74,4 \pm 7,2]$ under hypercapnic stimuli was used in this study. A Mann-Whitney test with significance level set to 5\%, performed for all anthropometric variables, did not show any statistically significant differences between the subjects. No subject had known disease of the cardiovascular or respiratory system or any impairment of muscular or skeletal mobility. None was taking medication regularly. Flow signal was recorded with a pneumotacograph (Validyne-CD257) and low-pass filter with a cutoff frequency of $10 \mathrm{~Hz}$. Exhaled $\mathrm{CO}_{2}$ pressure was monitored by means of a capnometer (Hewlett Packard-47210A) and airflow, expired fraction of $\mathrm{O}_{2}\left(\mathrm{P}_{\mathrm{ETO}}\right)$ and $\mathrm{CO}_{2}\left(P_{E T C O}\right)$ signals were recorded with a sampling frequency of $100 \mathrm{~Hz}$.

In order to produce hypercapnic stimulation, each subject breathed through a mouthpiece a gas mixture with different $\mathrm{CO}_{2}$ concentrations. Subjects were seated in a comfortable position, wore a clip on the nose and were breathing through a mouthpiece connected to a low-resistance one-way valve. The inspiratory port of the valve was connected to an external $\mathrm{CO}_{2}$ source. The protocol began with a $\mathrm{CO}_{2}$ free mixture, then $\mathrm{CO}_{2}$ concentration in the inhaled flow was increased four times every three minutes. Thus, five levels of hypercapnia stimulus (including the free of $\mathrm{CO}_{2}$ mixture) were applied. Flow signal was processed to detect inspiration and expiration onsets during each cycle. For each level of $\mathrm{CO}_{2}$, measurements of $V_{T}, f_{R}, T_{I}$ and $T_{E}$ were obtained from the twenty most representative respiratory cycles of the flow signal after the first minute because it was considered an adaptation-interval in each level of hypercapnia [79].

$\dot{V}(t)$ and $V(t)$ waveforms for two experimental ventilation levels, rest and moderate 
hypercapnia, are shown in Fig. 4 Experimental data of $T_{I}$ and $V_{T}$ as a function of $\dot{V}_{E}$ are shown in the Fig. 5. Both Fig. 4 and Fig. 4 show that increases in the $P_{I C O}$ produced changes in $\dot{V}_{E}$, which were adjusted by both increasing $f_{R}$ and $V_{T}$ and decreasing $T_{I}$. These observations are in agreement with other studies [80, 81, 82].
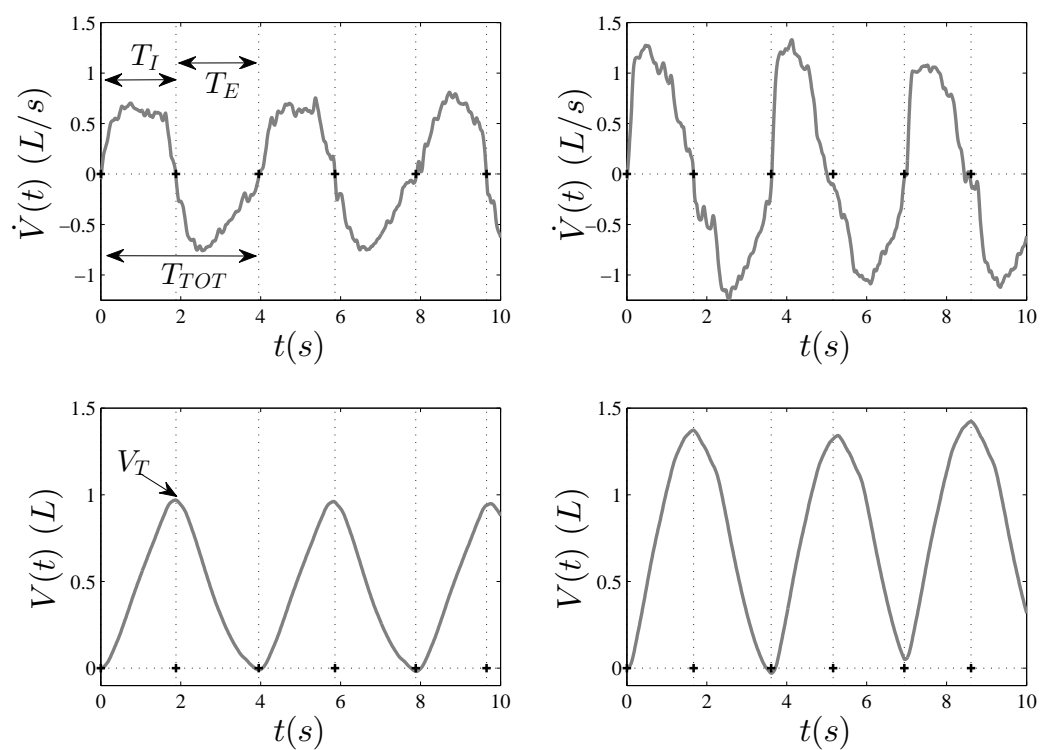

Figure 4: Flow $(\dot{V}(t))$ and volume $V(t)$ waveforms for basal $\dot{V}_{E}$ level (left) and moderate hypercapnia (right). Tidal volume $\left(V_{T}\right)$ and respiratory frequency $\left(f_{R}\right)$ increase at higher stimulus. $T_{T O T}, T_{I}$ and $T_{E}$ are total respiratory cycle, inspiratory and expiratory times respectively.

\subsection{Breathing pattern fitting}

The optimization of breathing pattern was performed by minimizing $J$ (Eq. 1) depending on five parameters included in the mathematical expressions of respiratory variables $x=\left[t_{1}, t_{2}, a_{1}, a_{2}, \tau\right]$ (Eqs. 7 and 9 ). In this model, $J$ is a nonlinear function which is subject to nonlinear constrainers and fixed boundaries in order to search a solution with physiological meaning. In this sense, the optimization problem statement was made by the following expression:

$$
\min \left\{J(x): c_{i}(x) \leq 0, i \in I ; \quad c_{j}(x)=0, j \in v\right\}
$$



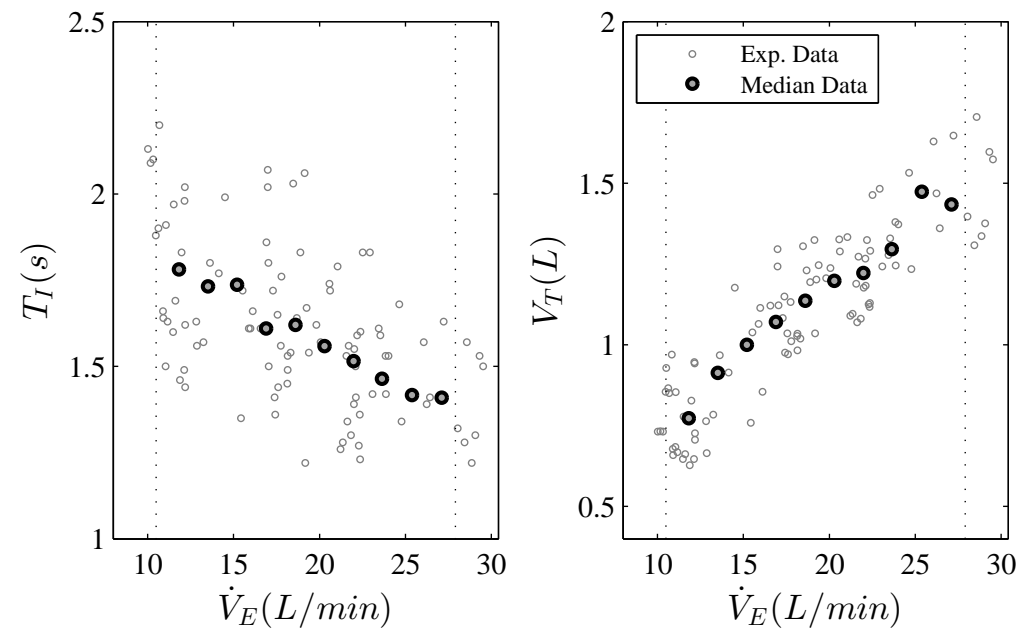

Figure 5: Experimental data of inspiratory time $\left(T_{I}\right)$ and tidal volume $\left(V_{T}\right)$ as a function of ventilation $\left(\dot{V}_{E}\right)$. Median values of $T_{I}$ and $V_{T}$ within 10 levels of $\dot{V}_{E}$, equidistantly distributed from $10 \mathrm{~L} / \mathrm{min}$ to $30 \mathrm{~L} / \mathrm{min}$, are also shown.

Each $c_{i}$ and $c_{j}$ is evaluated in the parameter space $R^{n}$ defined by $x(n=5) . I$ and $v$ denote constraints formed by inequalities and equalities respectively. In this case, inequality constraints have the form $c(x) \leq 0$, where $c$ is a vector of constraints, one component for each constraint, and were represented by the following boundary conditions:

$$
\begin{gathered}
c_{1}(x)=-x+c_{1} \\
c_{2}(x)=x+c_{2} \\
c_{3}(x)=-J(x)
\end{gathered}
$$

Where $c_{1}=[0.5,0.5,3.0,-20,0.15]$ and $c_{2}=[6,6,80,0.5,1.20]$ denote lower and upper bound of $x$, respectively. The inequality constraints are accomplished when each $c_{i}(x) \leq 0$. In addition, solutions found after optimization were assessed to guarantee that they were located within the search space and not in the bounds. The constraint $c_{3}$ was formulated in order to assure that $J(x)$ be positive according to a physiological interpretation. In addition, equality constraints were represented by the 
following expressions:

$$
\begin{array}{r}
c_{4}(x)=V\left(t_{1}+t_{2}\right)-V(0) \\
c_{5}(x)=V\left(T_{I}\right) \\
c_{6}(x)=V\left(t_{1}+t_{2}\right)
\end{array}
$$

where the first term of Eq. 18 establishes that inspired and expired tidal volume must be equal whereas the other elements determine that the airflow at the end of both inspiration and expiration must be zero respectively.

Fig. 6 describes the algorithm developed to find the optimal vector $x\left(x_{o p t}\right)$. In order to be easier the assessing of optimization algorithms at this level of optimization, the parameters $\lambda_{1}, \lambda_{2}$ and $n$ were set to 1 .
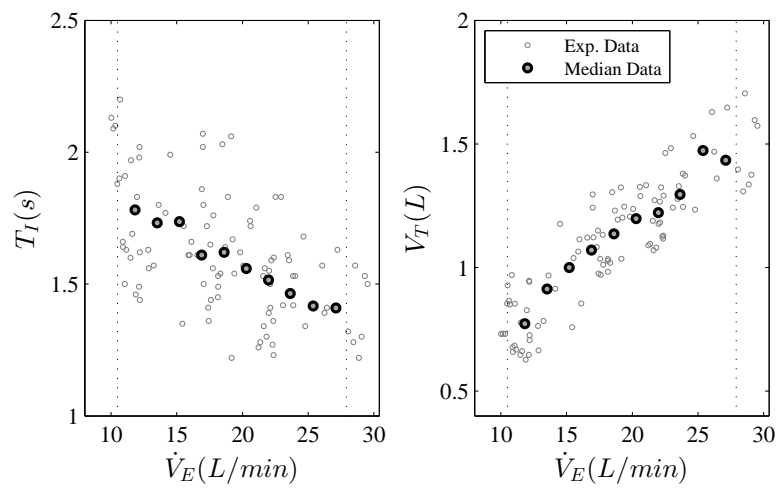

Figure 6: Diagram of the procedure to obtain the optimal vector of parameters $x$ that minimizes respiratory $\operatorname{cost}(J)$.

Due to $T_{I}$ could be higher than $t_{1}$ (neural inspiratory time), $T_{I}$ was determined at the time when the airflow changes from positive to negative and $T_{E}$ was calculated from the difference between the cycle period $\left(T_{T O T}=t_{1}+t_{2}\right)$ and $T_{I}$. Tidal volume, $V_{T}$, was determined by the value $V\left(T_{I}\right)$.

Finally, the solution of $x$ was obtained minimizing the cost function $J$ and using the two measures to quantify mechanical WOB (Eqs. 4a or Eqs. 5a) presented in [9]. Then, their results were compared with the experimental data in order to evaluate which measure is more appropriate to model the physiological respiratory system. 


\subsection{Model fitting to experimental data}

Model fitting was performed searching the parameter vector $\theta=\left[\lambda_{1}, \lambda_{2}, n\right]$ by adjusting the model response to experimental data. Parameters $\lambda_{1}$ and $\lambda_{2}$ are weights of inspiratory and expiratory work, respectively, and $n$ is the efficiency parameter of mechanical plant displayed in Eqs. 4a and 5a In this case, it is important to note that optimization of $\theta$ involves a simultaneous breathing pattern optimization by the minimization of $J$ (Eq. 11) presented in the previous subsection.

The optimum vector $\theta$ was obtained by minimizing prediction error $(P E)$ between the model response and experimental data, as follows:

$$
P E(\%)=\frac{1}{2 k} \sum_{\text {var }=T_{I}, V_{T}} \sum_{i=1}^{k}\left|\frac{\operatorname{var}_{E X P}(i)-\operatorname{var}_{S I M}(i)}{\operatorname{var}_{S I M}(i)}\right| \times 100 \%
$$

where $\operatorname{var}_{E X P}$ and $\operatorname{var}_{S I M}$ correspond to experimental and simulated variables respectively, and $k$ is the number of samples considered along $\dot{V}_{E}$. This optimization problem was formulated by the following expression:

$$
\min \left\{P E(\theta): \operatorname{pec}_{i}(k) \leq 0, k \in I\right\}
$$

where $p e c$ represent the prediction error constraints and each $p e c_{k}$ is evaluated in the parameter space $R^{n}$ defined by $\theta(n=3)$. I denotes constraints formed by inequalities. The search space of this optimization problem was built by using the next inequality constraints:

$$
\begin{aligned}
& \operatorname{pec}_{1}(x)=-x+\operatorname{pec}_{1} \\
& \operatorname{pec}_{2}(x)=+x+\operatorname{pec}_{2}
\end{aligned}
$$

Where $p e c_{1}=[0,0,0]$ and $p e c_{2}=[5.0,1.5,5.0]$ denote lower and upper bound of $\theta$ respectively. Like in breathing pattern optimization, the inequality constraints are accomplished when each $\operatorname{pec}_{k}(x) \leq 0$. In addition, solutions found after optimization were assessed to guarantee that they were located within the search space and not in the bounds. The algorithm developed to find the optimum vector $\theta\left(\theta_{\text {opt }}\right)$ can be describe by the following steps (see Fig.77): 


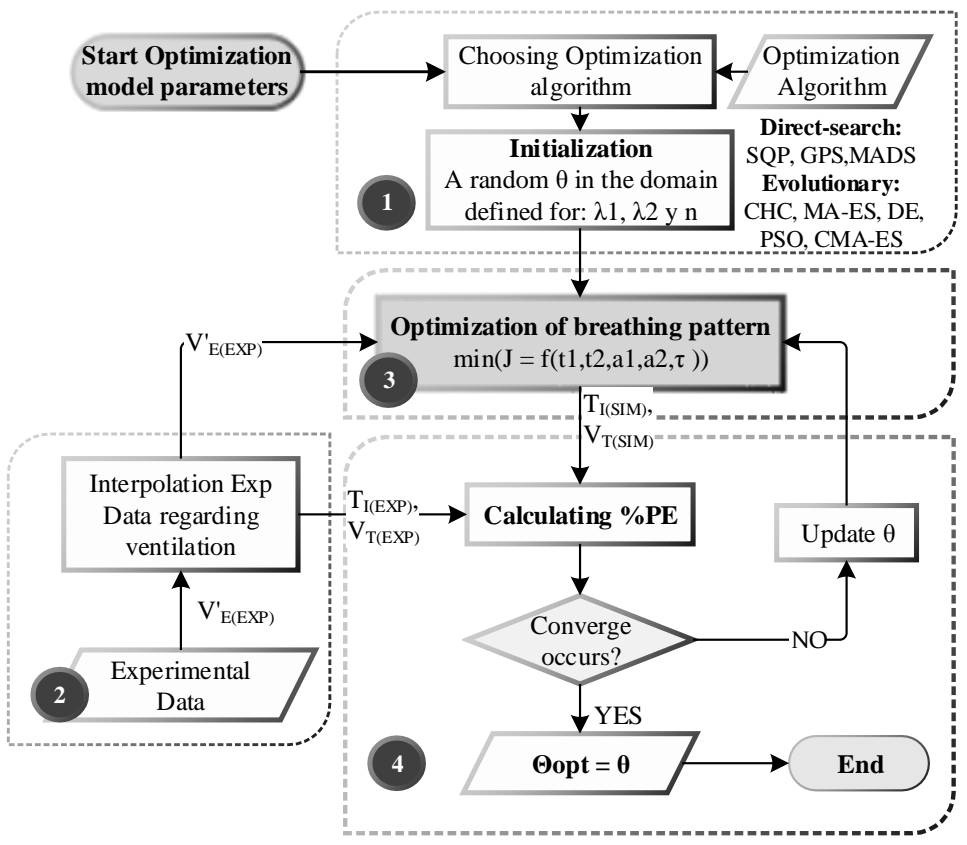

Figure 7: Diagram of the procedure to obtain the optimal vector of parameters $\theta_{\text {opt }}$ that adjusts the breathing pattern depending on minimizing respiratory cost $(J)$. The procedure is composed by four steps (see text)

- Selection of an optimization algorithm to adjust the model parameters to experimental data and randomly initialization of within the domain defined for $\lambda_{1}, \lambda_{2}$ and $n$.

- Loading experimental data. In this step, ten values of $\dot{V}_{E(E X P)}$ between 10 and $30 \mathrm{~L} / \mathrm{min}$ were considered. Then, in order to get uniformly distributed data in all subjects, experimental data of $T_{I}\left(T_{I(E X P)}\right)$ and $V_{T}\left(V_{T(E X P)}\right)$ were interpolated in function of $\dot{V}_{E(E X P)}$.

- Optimization of breathing pattern by adjusting five model parameters $\left(t_{1}, t_{2}, a_{1}, a_{2}, \tau\right)$ which minimize the cost function $\mathrm{J}$ (see Fig. 6). Levels of $\mathrm{PICO}_{2}$ associated with the $\dot{V}_{E(E X P)}$ considered in the previous step were used for this optimization. Then, simulated values of $T_{I}$ and $V_{T}\left(T_{I(S I M)}\right.$ and $\left.T_{E(S I M)}\right)$ were obtained.

- Optimization of the vector $\theta$ by minimizing $P E$ (Eq. 20p which was calculated from simulated and experimental data for $T_{I}$ and $V_{T} . \theta$ was iteratively updated 
according to the searching procedure of the optimization algorithm selected in the step 1 . The iterative process ended obtaining $\theta_{\text {opt }}$ when the maximum number of function evaluations was reached.

\subsection{Statistical analysis}

Wilcoxon-Mann-Whitney non-parametric test (WMW) was used to identify statistical differences between the found solutions with a significance level $\alpha=0.05$.

The validation of values from vector $\theta$ was performed using the leaving-one-out technique (LOO). In this case, the vector $\theta$ was adjusted eight times leaving one subject of study population out for each iteration and using it, in turn, to calculate $P E$. As result, eight vectors of $\theta$ and $P E$ were obtained for each model. Differences among the eight vectors of $\theta$ were evaluated using the Friedman test. Non-significant differences would mean that the found vectors $\theta$ are statistically similar and there is no significant dependence on the subject left out during optimization process.

In order to quantify differences between experimental data and results for each WOB estimation, $P E$ and also relative mean square error were calculated by using Eq. 20 and the following expression:

$$
R M S E=\frac{1}{2 k} \sum_{v a r=T_{I}, V_{T}} \sum_{i=1}^{k}\left(\frac{\operatorname{var}_{E X P}(i)-\operatorname{var}_{S I M}(i)}{\operatorname{var}_{S I M}(i)}\right)^{2}
$$

\section{Results}

\subsection{Breathing pattern fitting}

The values of $J$ resulting obtained from simulations of RSM1 and RSM2 with the eight analyzed optimization algorithms as a function of number of evaluations $(N)$ are shown in Fig. 8 It can be seen that the lowest $J$ and its corresponding value $N$ were obtained by SQP in both models. Statistical differences were found between SQP and the others algorithms using WMW $(p-$ value $<0.001$ ). Thus, this algorithm was statically more appropriated for the optimization of breathing pattern, so it was selected to adjust breathing pattern during model fitting to experimental data. 

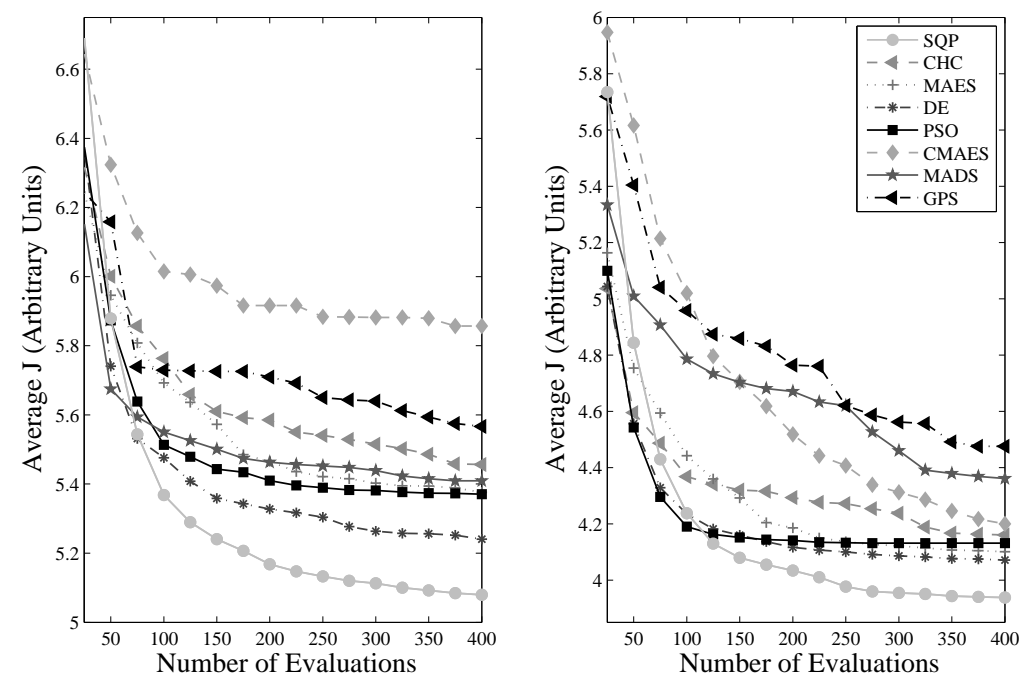

Figure 8: Respiratory cost values ( $J$ ) of RSM1 (left) and RMS2 (right) under hypercapnia stimuli by using eight optimization algorithms. $J$ is shown as a function of number of evaluations. Average values of $J$ were calculated from 25 runs carried out with each algorithm.

\subsection{Model fitting to experimental data}

Fig. 9 shows PE obtained by using the eight optimization algorithms under study in function of number of evaluations $(N)$. Tab. 3 presents the mean and standard deviation values of PE reached at the maximum number of evaluations for both RSM1 and RSM2.

In this case, the best performance associated with the lowest PE and standard deviation was obtained with the algorithm CMA-ES for both RSM1 and RSM2. Statistically significant differences were identified between CMA-ES and the other algorithms ( $p$-value $<0.0001)$. Fig. 10 shows simulated and experimental values of $T_{I}$ and $V_{T}$ as a function of $\dot{V}_{E}$ for each model using CMA-ES. It can be seen that RSM2 has a better fit to the median values of experimental data than RSM1.

Tab. 4 shows mean and standard deviation values of $\lambda_{1}, \lambda_{2}$ and $n$ obtained with LOO and CMA-ES algorithm. Differences among these eight values were not statistically significant using the Friedman test in any of the parameters and in both model ( $p$ value $=$ n.s.). 

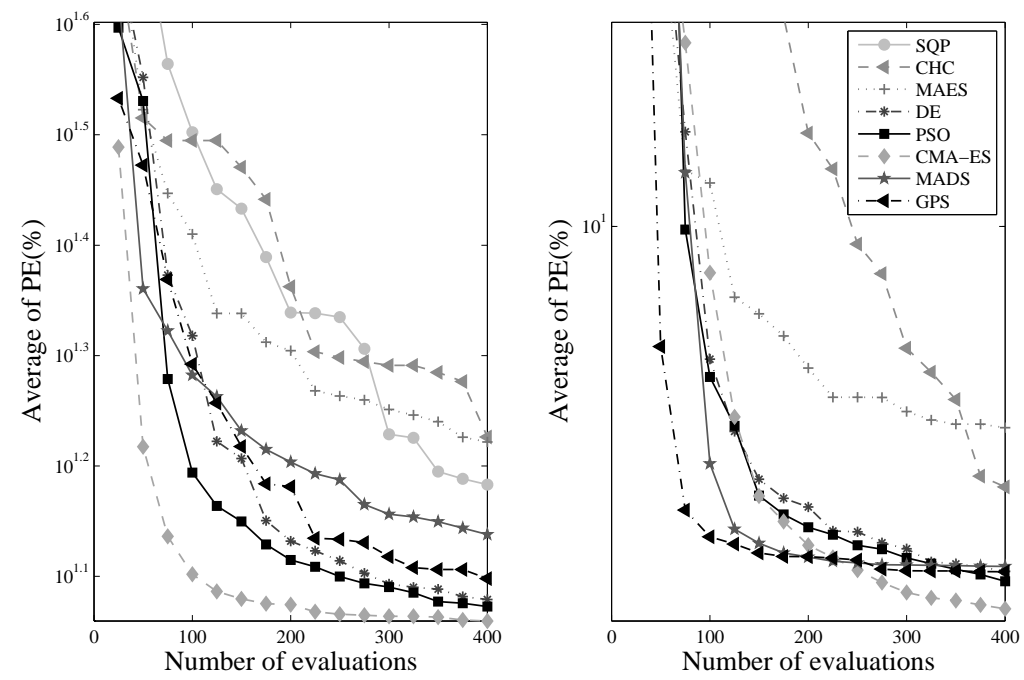

Figure 9: Prediction error values (\%PE) as a function of number of evaluations for RSM1 (left) and RSM2 (right) using eight optimization algorithms. Average values of $\% P E$ were calculated from 25 runs carried out with each algorithm.
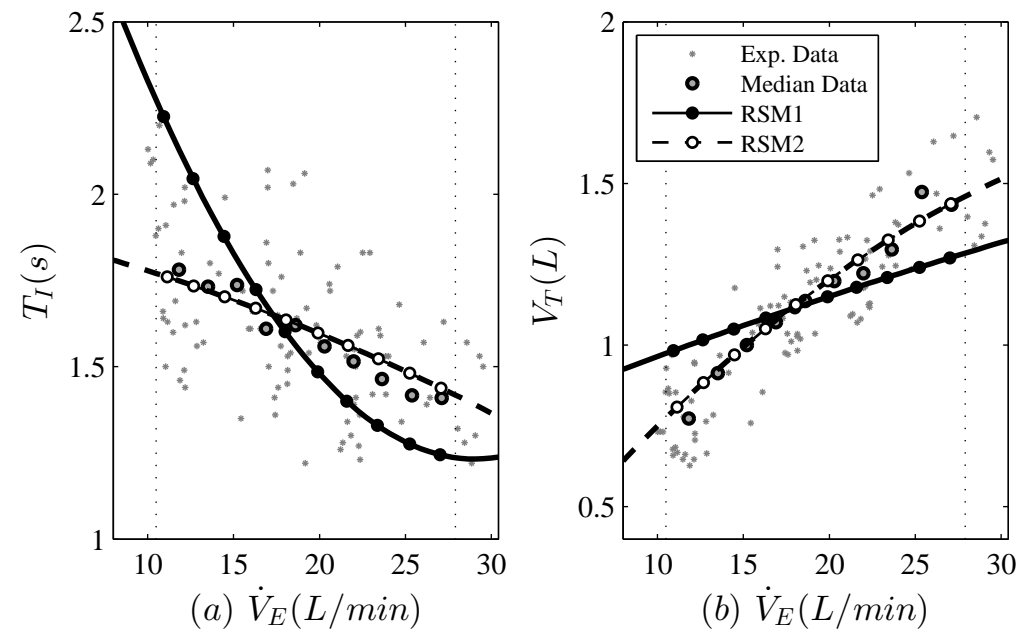

Figure 10: Experimental data and simulation results of RSM1 and RSM2. (a) Inspiratory time ( $\left.T_{I}\right)$ and (b) tidal volume $\left(V_{T}\right)$ as a function of ventilation $\left(\dot{V}_{E}\right)$.

In addition, prediction and relative mean square errors (\%PE and \%RMSE respectively) of $T_{I}$ and $V_{T}$ using CMA-ES algorithm are presented in Table 5 . The lowest val- 
Table 3: Prediction error (\%PE) in mean and standard deviation obtained by using eight algorithms during optimization process. Each algorithm was run 25 times.

\begin{tabular}{lll} 
Model & Algorithm & $\% \mathrm{PE}($ mean $\pm \sigma)$ \\
\hline & SQP & $15.589 \pm 4.512$ \\
& CHC & $16.718 \pm 4.797$ \\
& MAES & $16.792 \pm 3.469$ \\
RSM1 & DE & $11.998 \pm 0.378$ \\
& PSO & $11.958 \pm 0.619$ \\
& CMA-ES & $11.443 \pm 0.239$ \\
& MADS & $13.730 \pm 2.331$ \\
& GPS & $12.530 \pm 1.014$ \\
\hline SQP & $12.792 \pm 3.333$ \\
CHC & $9.384 \pm 0.157$ \\
MAES & $9.484 \pm 0.365$ \\
DE & $9.212 \pm 0.031$ \\
PSO & $9.183 \pm 0.063$ \\
CMA-ES & $9.121 \pm 0.021$ \\
MADS & $9.234 \pm 0.121$ \\
GPS & $9.185 \pm 0.131$ \\
\hline
\end{tabular}

ues in RMS2 showed a better fit to experimental data, with an improvement of $32.4 \%$ and $72.4 \%$ with respect to RSM1 respectively ( $p$-value $<0.04$ and $p$-value $<0.03$ ). 
Table 4: Optimal model parameters found by using CMA-ES algorithm in RSM1 and RSM2 (mean and standard deviation from the solutions obtained by Leaving-One-Out method

\begin{tabular}{lccc} 
& \multicolumn{3}{c}{ Optimal parameters } \\
Model & $\lambda_{1}$ & $\lambda_{2}$ & $n$ \\
& - & $0.86 \pm 0.02$ & $0.00 \pm 0.00$ \\
RSM1 & & & \\
RSM2 & $4.90 \pm 0.13$ & $0.74 \pm 0.07$ & $1.27 \pm 0.07$ \\
\hline
\end{tabular}

Table 5: Prediction error (\%PE) and relative mean square error (RMSE) by using CMA-ES algorithm in RSM1 and RSM2 (mean and standard deviation from the solutions obtained by leaving-one-out method)

\begin{tabular}{ccccc} 
Error & Model & $T_{I}$ & $V_{T}$ & Average \\
\hline & RSM1 & $14.36 \pm 5.44$ & $13.53 \pm 4.20$ & $13.94 \pm 4.62$ \\
PE(\%) & RSM2 & $9.75 \pm 3.44$ & $9.09 \pm 3.59$ & $9.42 \pm 3.42$ \\
\hline \multirow{2}{*}{ RMSE } & RSM1 & $2.15 \pm 1.30$ & $1.91 \pm 1.32$ & $2.03 \pm 1.16$ \\
& RSM2 & $0.44 \pm 0.33$ & $0.69 \pm 0.53$ & $0.56 \pm 0.36$ \\
\hline
\end{tabular}

\section{Discussion and Conclusion}

\subsection{Optimization problem}

Two approaches for the respiratory control system modeling based on simultaneous optimization of ventilation and breathing pattern, which were called in this study RSM1 and RSM2, have been analyzed [9]. Such optimization was performed by minimizing a respiratory cost function $(J)$ that reflects the balance of chemical $\left(J_{c}\right)$ and mechanical $\left(J_{m}\right)$ costs of breathing. Differences between both approaches were determined by the equations proposed to quantify $J_{m}$ during inspiratory and expiratory phases (Eqs. 4a, 5a, 4b and 5b respectively), which in turn represent estimates of the mechanical work of breathing (WOB).

Although in this model, two equation sets were proposed to quantify the mechanical cost, RSM2 was discarded in [9] because it always led to an impulsive inspiratory 
pressure profile with an extremely small inspiratory duty cycle; this behavior had been also noted by other researchers [83, 75]. However, in this study with the used optimization techniques was possible to adjust the model parameters in order to obtain a response with a realistic behavior (see Fig. 3 and Fig. 10).

The fitting of model parameters involved two nested optimizations: the optimization of breathing pattern by fitting $x=\left[t_{1}, t_{2}, a_{1}, a_{2}, \tau\right]$ and the adjustment of model responses to experimental data by fitting $\theta=\left[\lambda_{1}, \lambda_{2}, n\right]$. The former was performed breath to breath by minimizing $J$, whereas the latter was performed by minimizing $P E$ in all populations.

For the first optimization, SQP showed the best performance: the lowest respiratory cost $(J)$ and the highest convergence speed. Additionally, SQP allowed easily implementing multiple non-linear constraints through mathematical expressions, whereas evolutionary algorithms required defining a complex search space according to model constraints. Because this optimization is carried out each breathing cycle, using an optimization algorithm with a high convergence speed is decisive to improve the model performance. For the second optimization, CMA-ES showed the best performance associated with the lowest PE(\%) in both RSM1 and RSM2, whereas SQP was the worse (see Fig. 9). Graphical representations of $\operatorname{PE}(\%)$ vs. [ $\left.\lambda_{2}, \mathrm{n}\right]$ for RSM1 (see Fig. 11) and PE(\%) vs. $\left[\lambda_{1}, \lambda_{2}, \mathrm{n}\right]$ for RSM2 (see Fig. 12) allowed to visualize the complexity of both landscape estimates and to find the reason of different performances found among algorithms. In both cases, the surfaces were shaped considering all data base.

PE(\%) surface of RSM1 (see Fig. 11) presented roughness around the optimum value. This favors the algorithms which are more robust to noise like evolutionary algorithms, because their main search strategies do not make any assumption about the underlying fitness landscape. For this reason, CMA-ES, PSO and DE showed better performances and solution/time relationship than direct-search algorithms (see left graphic of Fig. 9]. By contrary, PE(\%) surface of RSM2was smoother around the optimal value (see Fig. 12, so strategies like GPS and MADS were faster to find a solution (see right graphic of Fig.99).

The performance found in the optimization algorithms can be explained taking into account their main searching properties. On one hand, a characteristic of direct-search 


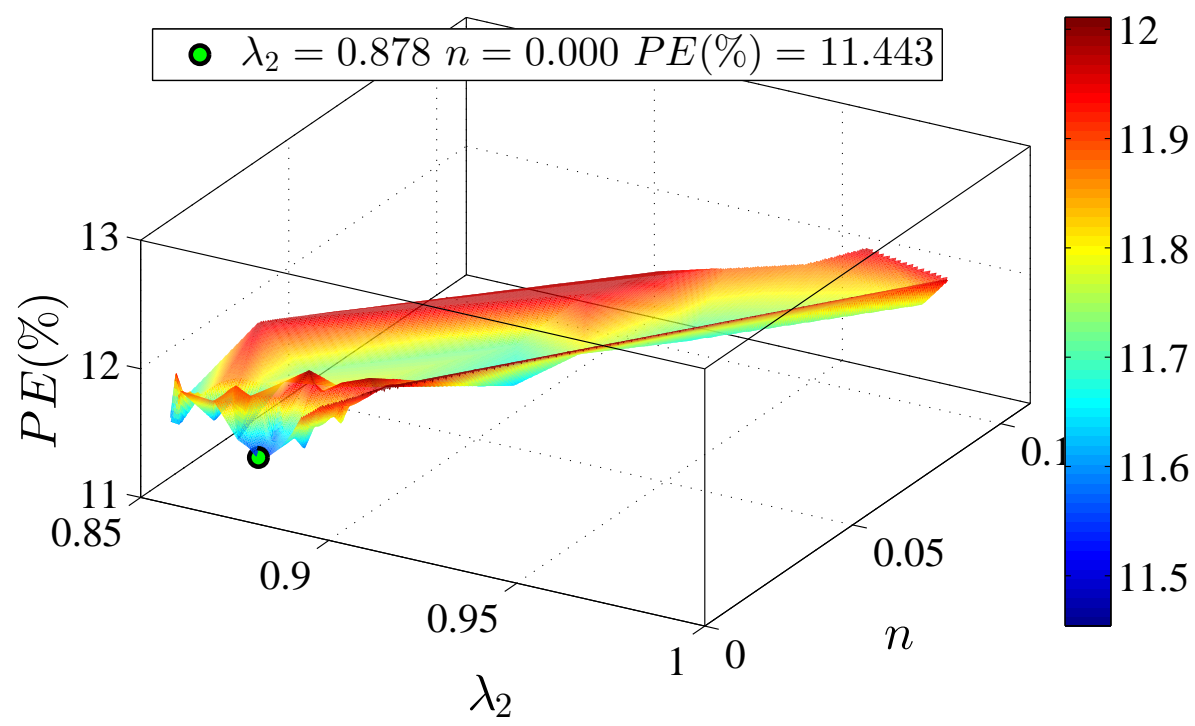

Figure 11: Topology of cost function for prediction error $(P E(\%))$ of RSM1 as a function of model parameters, $\lambda_{2}$ and $n$

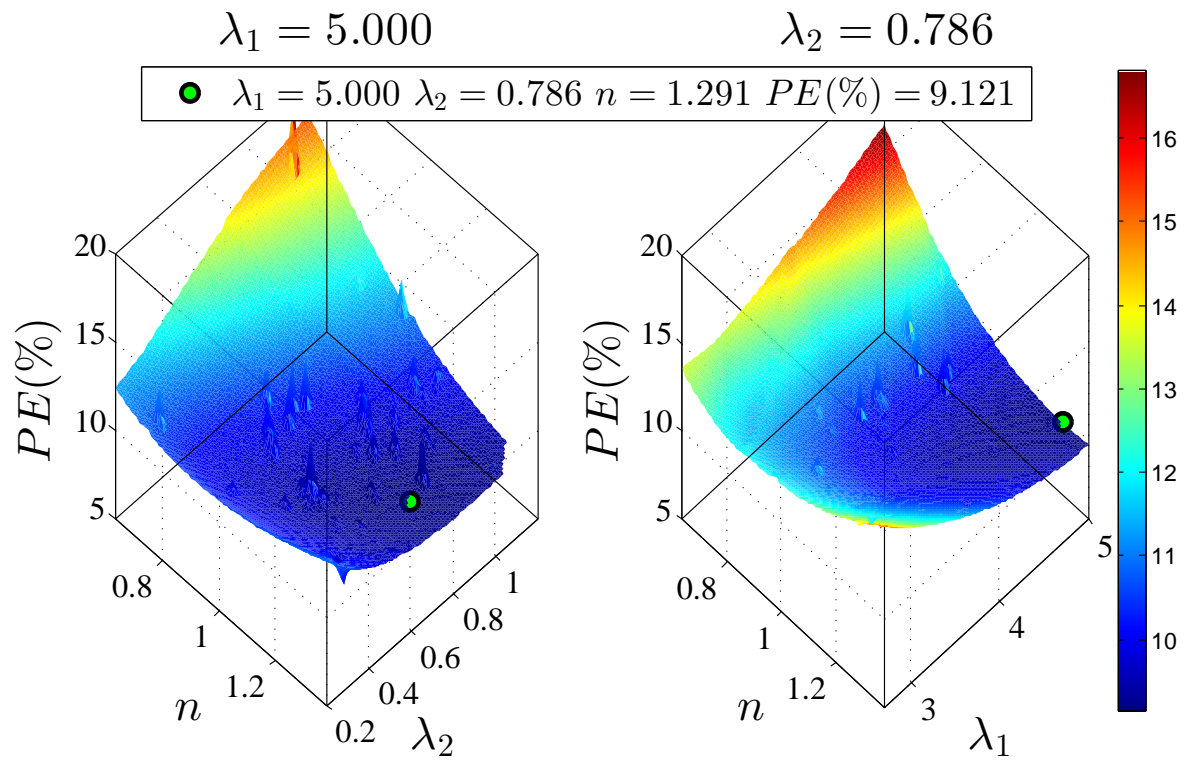

Figure 12: Topology of cost function for prediction error (PE(\%)) of RSM2 as a function of model parameters: $n$ and $\lambda_{2}$ (left) and $n$ and $\lambda_{1}$ (right) 
(or deterministic) methods is that they capture very fast the right path to the nearest optimum, independently on being a local or global algorithm search. However, after locating the area around this optimum it might oscillate until all constraints are satisfied and, in this case, small constraint violation often slow down the convergence rate of the method. On the other hand, evolutionary algorithms proceed with slower rate, due to their random search, but the absence of strict mathematical rule, which govern the convergence rate of the mathematical program methods, make evolutionary algorithm less vulnerable to local optima and therefore it is likely to convergence towards the global optimum in non-convex optimization methods [84, 16].

Finally, regarding the quality of the solution $\theta_{o p t}$, CMA-ES had the best performance in the parameter adjustment. This algorithm permitted to find model parameters, statistically significant, with the lowest PE and standard deviation showing the best fitting of both respiratory control system models with respect to experimental data. This performance was obtained possibly due to CMA-ES is an evolutionary algorithm that is able to optimize functions without making strong assumptions about them. It also has several other advantages like it does not require to perform extensive parameter setting; its standard values have shown to work well in many different contexts [57].

\subsection{Physiological meaning}

Physiological meaning of model structure and its parameters have also a great relevance. RSM1 and RSM2 were formulated based on the minimization WOB and the prevention of damages in the mechanical plant in order to take advantages from those

models in devices and new therapies development. Regarding RSM1, Eqs. 4a and 4b represent a classical measurement of the mechanical work rate for both inspiration and expiration: an integral of product of driving pressure, $P(t)$, and airflow, $\dot{V}(t)$. It can note that polarity of $J_{m}$ during expiration is negative because $\dot{V}(t)<0$ and $P(t)>0$. Although there are good reasons to assume that during inspiratory phase the oxygen consumption of muscles is roughly proportional to product of $P(t)$ and $\dot{V}(t)$ (like in Eq. 4a, this approach can be not reasonable for the expiration because at the beginning of a normal expiration the inspiratory muscles continue their action by opposing the expiration and performing an inverse work with an oxygen consumption that, even 
though could be smaller than inspiratory work [85], implies a waste of energy that is not described by the Eq. $4 \mathrm{~b}$

On the other hand, RSM2 establishes that $J_{m}$ during inspiration is a weighting sum by the parameter $\lambda_{1}$ of the inspiratory pressure integral, $P(t)$, and the average square magnitude of volume acceleration, $\ddot{V}$ (see Eq. 5a. In this equation, the former represents a measure of the oxygen cost of breathing during an isometric contraction and the latter penalizes fast changes in the airflow rate. During expiration, $J_{m}$ also depends on uniformity of expiratory flow (Eq. $5 \mathrm{~b}$ ), therefore, unlike of RSM1, $J_{m}$ is always positive and its physiological meaning would be more suitable. The term $\ddot{V}$ in both inspiratory and expiratory phases avoids decreases in the efficiency of muscle contraction, due to high acceleration and high shortening velocities, and therefore harmful effects [24].

In both models (Eqs. $4 \mathrm{a}$ and $5 \mathrm{a}$ ), $\xi_{1}$ and $\xi_{2}$ quantify mechanical efficiency of respiratory muscles and parameter $n$ remarks nonlinear effect of those efficiencies in WOB. Whereas in RSM1 $n$ is equal to 0 (maximum efficiency), in RSM2 the obtained value for $n$ is 1.26 . The null value obtained in RSM1 implies the exclusion of efficiency factors from Eq. $4 \mathrm{a}$.

Regarding the values of parameter $\lambda_{2}$, these were quite similar and lower than unity in both models (see Tab. 4), however the polarity of $J_{m}$ in RSM2 makes sense to describe the mechanical cost during expiration [6]. In addition, the obtained value for $\lambda_{1}$ in RSM2 (see Eq. 5a highlights the importance of taking into account the volume acceleration in the inspiratory $J_{m}$ when subjects with increased ventilatory demand are simulated.

In [9], $\lambda_{2}=1$ and $n=3$ were used as nominal values and larger values of $n$ were related to inspiratory resistive loads (IRL) where the efficiency factors $\xi_{1}$ and $\xi_{2}$ are significant. $\lambda_{2}$ was conveniently chosen such that values of $t_{2}$ and $P\left(t_{1}\right)$ were comparable to experimental values normally seen in human subjects and $n$ was adjusted to experimental data of hypercapnic ventilatory responses of subjects under IRL. These values were not used in this study due to nature of experimental data and the final aim of this study: validate the model by using experimental data of health subjects under hypercapnia. On the other hand, although several researchers [43, 38, 39, 40] have used this model, none of them has provided information about parameter values employed 
in their simulations.

The values of PE obtained for RSM1 and RSM2 were $13.94 \%$ and $9.42 \%$, respectively. Taking into account the experimental data dispersion (8.99\%), a very good fitting of RSM2 to experimental data can be concluded. Finally, although RSM1 has been more used in the literature than RSM2 [38, 39, 40], because of its prediction capability and also its physiological meaning, the latter has been found more suitable to simulate the respiratory system response under ventilatory stimuli such as hypercapnia. Thus, this is more suitable to be used in further simulations of respiratory pathologies or assisted ventilation.

A possible limitation of the present study is related to the sample size. For this reason, it is expected to extent the results with more subjects in future studies in order to confirm that the analyzed group is representative enough and also to confirm the present results and conclusions. However, differences obtained among estimates RSM1 and RSM2 by using the analyzed optimization algorithms were statistically significant, the effect size statistics which depends on the sample size was taken into account, and size of the population was the same as that employed in studies related to physiological system, among them the respiratory system, [86, 87, 88]. For these reasons, we can conclude that these results are promising to provide a realistic estimate and, therefore, can be relevant and of interest.

\section{Acknowledgment}

This study was partially funded by the Spanish government MINECO (DPI2014J01682), the Technical University of Catalonia (FPU-707.707), the University of Antioquia - Colombia (CODI-E01539-MDC11-1-07). Authors want to thank the Unitat de Semicrítics of Hospital de la Santa Creu i Sant Pau, led by Dr. Salvador Benito, for its help in designing experimental protocol and in signal recording.

\section{References}

[1] L. Cheng, O. Ivanova, H.-H. Fan, M. C. K. Khoo, An integrative model of respiratory and cardiovascular control in sleep-disordered breathing, Central Cardiores- 
piratory Regulation: Physiology and Pathology 174 (1-2) (2010) 4-28.

[2] S. Ganzert, J. Guttmann, D. Steinmann, S. Kramer, Equation Discovery for Model Identification in Ventilated Human Lung, Discovery (2010) 296-310.

[3] Z. L. Topor, M. Pawlicki, J. E. Remmers, A computational model of the human respiratory control system: responses to hypoxia and hypercapnia., Annals of biomedical engineering 32 (11) (2004) 1530-1545.

[4] F. T. Tehrani, Mathematical analysis and computer simulation of the respiratory system in the newborn infant, Biomedical Engineering, IEEE Transactions on 40 (5) (1993) 475-481.

[5] J. J. Marini, P. S. Crooke, A general mathematical model for respiratory dynamics relevant to the clinical setting, American Review of Respiratory Disease 147 (1) (1993) 14-24.

[6] C. Roussos, E. J. M. Campbell, Respiratory muscle energetics, Handbook of Physiology, Sect.3, Vol.3: Respiration 3 (PART 2) (1986) 481-509.

[7] W. Fincham, F. T. Tehrani, On the regulation of cardiac output and cerebral blood flow, Journal of Biomedical Engineering 5 (1) (1983) 73-75.

[8] J. J. Batzel, F. Kappel, D. Schneditz, H. T. Tran, Cardiovascular and respiratory systems : modeling, analysis, and control, Society for Industrial Mathematics, Philadelphia, PA, 2007.

[9] C. S. Poon, S. L. Lin, O. B. Knudson, Optimization character of inspiratory neural drive, J Appl Physiol 72 (5) (1992) 2005-2017.

[10] P. E. Gill, W. Murray, M. H. Wright, Practical optimization, Vol. 1, Academic press, 1981.

[11] P. E. Gill, W. Murray, M. A. Saunders, M. H. Wright, Procedures for optimization problems with a mixture of bounds and general linear constraints, ACM Transactions on Mathematical Software (TOMS) 10 (3) (1984) 282-298. 
[12] V. Torczon, Others, On the convergence of pattern search algorithms, SIAM Journal on optimization 7 (1) (1997) 1-25.

[13] R. M. Lewis, V. Torczon, Pattern search algorithms for bound constrained minimization, SIAM Journal on Optimization 9 (4) (1999) 1082-1099.

[14] J. E. Dennis, C. Audet, Mesh adaptive direct search algorithms for constrained optimization, SIAM Journal on optimization 17 (1) (2006) 188-217.

[15] L. Eshelman, The CHC adaptive search algorithm, in: G. Rawlins (Ed.), Foudations of Genetic Algorithms, Morgan Kaufmann, 1990, pp. 265-283.

[16] R. V. Sol, J. Marin, R. V. Sole, Macroevolutionary algorithms: A new optimization method on fitness landscapes, Evolutionary Computation, IEEE Transactions on 3 (4) (1999) 272-286.

[17] R. Storn, K. Price, Differential Evolution - A Simple and Efficient Heuristic for Global Optimization over Continuous Spaces, J. of Global Optimization 11 (4) (1997) 341-359.

[18] J. Kennedy, R. Eberhart, Particle swarm optimization, Neural Networks, 1995. Proceedings. IEEE International Conference on 4 (1995) 1942-1948.

[19] N. Hansen, A. Ostermeier, Completely Derandomized Self-Adaptation in Evolution Strategies, Evol. Comput. 9 (2) (2001) 159-195.

[20] C. S. Poon, Respiratory models and control, Biomedical Engineering Handbook 3 (1995) 2404-2421.

[21] J. Duffin, R. M. Mohan, P. Vasiliou, R. Stephenson, S. Mahamed, A model of the chemoreflex control of breathing in humans: model parameters measurement., Respiration physiology 120 (1) (2000) 13-26.

[22] J. Duffin, The role of the central chemoreceptors: a modeling perspective., Respiratory physiology \& neurobiology 173 (3) (2010) 230-43. 
[23] A. B. Otis, W. O. Fenn, H. Rahn, Mechanics of Breathing in Man, J Appl Physiol 2 (11) (1950) 592-607.

[24] R. P. Hamalainen, A. Sipila, Optimal control of inspiratory airflow in breathing, Optimal Control Applications and Methods 5 (2) (1984) 177-191.

[25] C. S. Poon, Ventilatory control in hypercapnia and exercise: optimization hypothesis, Journal of applied physiology 62 (6) (1987) 2447-2459.

[26] F. T. Tehrani, Optimal control of respiration in exercise, Engineering In Medicine And Biology 20 (6) (1998) 6-9.

[27] F. T. Tehrani, Function of brainstem neurons in optimal control of respiratory mechanics, Biological cybernetics 89 (3) (2003) 163-169.

[28] C.-S. S. Poon, C. Tin, Y. Yu, Homeostasis of exercise hyperpnea and optimal sensorimotor integration: the internal model paradigm, Respiratory physiology \& neurobiology 159 (1) (2007) 1-13.

[29] H. Li, W. M. Haddad, Optimal determination of respiratory airflow patterns using a nonlinear multi-compartment model for a lung-rib-cage system, in: American Control Conference (ACC), 2011, 2011, pp. 3524-3529.

[30] F. Rohrer, Physiologie der atembewegung, in: Handbuch der normalen u. pathol. Physiologie Bd. II, S, Springer, 1925, pp. 70-127.

[31] J. Mead, Control of respiratory frequency, J Appl Physiol 15 (3) (1960) 325-336.

[32] J. G. Widdicombe, J. A. Nadel, Airway volume, airway resistance, and work and force of breathing: theory, Journal of Applied Physiology 18 (5) (1963) 863-868.

[33] S. M. Yamashiro, F. S. Grodins, Optimal regulation of respiratory airflow., Journal of Applied Physiology 30 (5) (1971) 597-602.

[34] R. P. Hamalainen, A. A. Viljanen, A hierarchical goal seeking model of the control of breathing. Part I: Model Description, Biological cybernetics 29 (3) (1978) 151-158. 
[35] R. P. Hamalainen, A. A. Viljanen, A hierarchical goal seeking model of the control of breathing. Part II. Model performance, Biological cybernetics 29 (3) (1978) 159-166.

[36] C.-S. S. Poon, Optimal interaction of respiratory and thermal regulation at rest and during exercise: role of a serotonin-gated spinoparabrachial thermoafferent pathway., Respiratory physiology \& neurobiology 169 (3) (2009) 234-42.

[37] C.-S. S. Poon, Evolving paradigms in H+ control of breathing: from homeostatic regulation to homeostatic competition., Respiratory physiology \& neurobiology $179(2-3)(2011) 122-6$.

[38] T. Harada, H. Kubo, T. Mori, T. Sato, Pulmonary and cardiovascular integrated model controlled with oxygen consumption, in: Annual International Conference of the IEEE Engineering in Medicine and Biology - Proceedings, Vol. 7 VOLS, 2005, pp. 304-307.

[39] S.-L. Lin, N.-R. Guo, Simulation and comparative studies of dead space loading for human respiratory control under exercise and co inhalation, in: Proceedings of the IASTED International Conference on Modelling, Identification, and Control, MIC, 2012, pp. 279-285.

[40] R. Kinkead, R. Gulemetova, Neonatal maternal separation and neuroendocrine programming of the respiratory control system in rats., Biological psychology 84 (1) (2010) 26-38.

[41] B. G. Lindsey, I. A. Rybak, J. C. Smith, Computational models and emergent properties of respiratory neural networks, Comprehensive Physiology 2 (3) (2012) 1619-1670.

[42] S.-L. S.-1. Lin, H.-W. H.-w. Shia, Comparative Studies of the Optimal Airflows and Ventilation Settings under Continuous Resistive and Elastic Loadings, Journal of Computational Information Systems 13 (7) (2011) 4594-4601.

[43] L. Y. Serna, A. M. Hernandez, M. A. Mañanas, Computational tool for modeling and simulation of mechanically ventilated patients, in: 2010 Annual Inter- 
national Conference of the IEEE Engineering in Medicine and Biology Society, EMBC'10, 2010, pp. 569-572.

[44] Advanced closed loops during mechanical ventilation (PAV, NAVA, ASV, SmartCare), Best Practice and Research: Clinical Anaesthesiology 23 (1) (2009) 81-93.

[45] M. Karcz, A. Vitkus, P. J. Papadakos, D. Schwaiberger, B. Lachmann, State of the Art Mechanical Ventilation, Journal of cardiothoracic and vascular anesthesia 26 (3) (2011) 486-506.

[46] F. T. Tehrani, A control system for mechanical ventilation of passive and active subjects., Computer methods and programs in biomedicine 110 (3) (2013) 511-8.

[47] M. Wysocki, J. X. Brunner, Closed-loop ventilation: an emerging standard of care?, Critical care clinics 23 (2) (2007) 223-240.

[48] F. Lellouche, J. Mancebo, P. Jolliet, J. Roeseler, F. Schortgen, M. Dojat, B. Cabello, L. Bouadma, P. Rodriguez, S. Maggiore, M. Reynaert, S. Mersmann, L. Brochard, A multicenter randomized trial of computer-driven protocolized weaning from mechanical ventilation, American Journal of Respiratory and Critical Care Medicine 174 (8) (2006) 894-900.

[49] P. T. Boggs, J. W. Tolle, Sequential quadratic programming, Acta numerica 4 (1) (1995) $1-51$.

[50] A. Lambrou, H. Papadopoulos, A. Gammerman, Reliable Confidence Measures for Medical Diagnosis With Evolutionary Algorithms, Information Technology in Biomedicine, IEEE Transactions on 15 (1) (2011) 93-99.

[51] S. Ghosh, S. Maka, Genetic algorithm based $\{$ NARX $\}$ model identification for evaluation of insulin sensitivity, Applied Soft Computing 11 (1) (2011) 221-226.

[52] S. E. Selvan, C. C. Xavier, N. Karssemeijer, J. Sequeira, R. A. Cherian, B. Y. Dhala, Parameter Estimation in Stochastic Mammogram Model by Heuristic Optimization Techniques, Information Technology in Biomedicine, IEEE Transactions on 10 (4) (2006) 685-695. 
[53] S. Ghosh, A differential evolution based approach for estimating minimal model parameters from IVGTT data, Computers in Biology and Medicine 46 (2014) 51-60.

[54] S. Damas, J. Santamar, O. Cordón, J. Santamaría, Feature-based image registration by means of the CHC evolutionary algorithm, Image and Vision Computing 24 (5) (2006) 525-533.

[55] M. C. Lee, S. J. Nelson, Supervised pattern recognition for the prediction of contrast-enhancement appearance in brain tumors from multivariate magnetic resonance imaging and spectroscopy., Artificial intelligence in medicine 43 (1) (2008) 61-74.

[56] V. Moscardó, P. Rossetti, F. J. Ampudia-Blasco, J. Bondia, Modelling adrenaline secretion during counterregulatory response in Type 1 Diabetes for improved hypoglycaemia prediction, IFAC-PapersOnLine 48 (20) (2015) 213-218.

[57] R. Aler, I. M. Galván, J. M. Valls, Applying evolution strategies to preprocessing EEG signals for braincomputer interfaces, Information Sciences 215 (2012) 5366.

[58] A. Das, Z. Gao, P. Menon, J. Hardman, D. Bates, Optimization of Mechanical Ventilator Settings, in: th 18th IFAC World Congress, Vol. 18, 2011, pp. 1425914264.

[59] Y. D. Zhang, S. H. Wang, G. L. Ji, Z. C. Dong, Genetic Pattern Search and Its Application to Brain Image Classification, Mathematical Problems in Engineering 2013.

[60] W. Yang, J. a. Feinstein, A. L. Marsden, Constrained optimization of an idealized Y-shaped baffle for the Fontan surgery at rest and exercise, Computer Methods in Applied Mechanics and Engineering 199 (33-36) (2010) 2135-2149.

[61] S. D. Ros, G. Colusso, T. A. Weschenfelder, L. de Marsillac Terra, F. de Castilhos, M. L. Corazza, M. Schwaab, A comparison among stochastic optimization 
algorithms for parameter estimation of biochemical kinetic models, Applied Soft Computing 13 (5) (2013) 2205-2214.

[62] M. K. Ahirwal, A. Kumar, G. K. Singh, Adaptive filtering of EEG/ERP through noise cancellers using an improved PSO algorithm, Swarm and Evolutionary Computation 14 (2014) 76-91.

[63] C. Zimmer, S. Sahle, Comparison of approaches for parameter estimation on stochastic models: Generic least squares versus specialized approaches, Computational Biology and Chemistry 61 (2016) 75-85.

[64] S. Koziel, X.-S. Yang, Computational optimization, methods and algorithms, Vol. 356, Springer, 2011.

[65] H. G. Beyer, H. P. Schwefel, Evolution strategies: A comprehensive introduction, Natural computing 1 (1) (2002) 3-52.

[66] R. M. Lewis, V. Torczon, M. W. Trosset, Direct search methods: then and now, Journal of Computational and Applied Mathematics 124 (1-2) (2000) 191-207.

[67] G. A. Jayalakshmi, S. Sowmyalakshmi, R. Rajaram, A Hybrid CHC Genetic Algorithm for Macro Cell Global Routing, Advances in soft computing: engineering design and manufacturing (2003) 343.

[68] E. Mezura-Montes, J. Velázquez-Reyes, C. A. Coello Coello, A comparative study of differential evolution variants for global optimization, in: Proceedings of the 8th annual conference on Genetic and evolutionary computation, GECCO '06, ACM, 2006, pp. 485-492.

[69] A. M. Sutton, D. Whitley, M. Lunacek, A. Howe, PSO and multi-funnel landscapes: how cooperation might limit exploration, in: Proceedings of the 8th annual conference on Genetic and evolutionary computation, GECCO '06, ACM, 2006, pp. $75-82$.

[70] A. Auger, N. Hansen, A restart CMA evolution strategy with increasing population size, in: Evolutionary Computation, 2005. The 2005 IEEE Congress on, Vol. 2, IEEE, 2005, pp. 1769-1776 Vol. 2. 
[71] K. Price, R. Storn, Differential Evolution Source Code (2012).

URL http://www.icsi.berkeley.edu/\{ \}storn/code.html

[72] B. Donckels, Optimization Algorithms (2012).

URL http://biomath. ugent.be/\{ \}brecht/downloads.html

[73] N. Hansen, CMA Evolution Strategy Source Code (apr 2012).

URL http://wWw.lri.fr/\{ $\}$ hansen/cmaesintro.html

[74] J. Marín Sanchéz, CHC and MA-ES Source Codes (2012).

URL http://sci2s.ugr.es/eamhco

[75] M. Younes, W. Riddle, A model for the relation between respiratory neural and mechanical outputs. I. Theory, J Appl Physiol 51 (4) (1981) 963-978.

[76] A. Anzueto, F. Frutos-Vivar, A. Esteban, I. Alía, L. Brochard, T. Stewart, S. Benito, M. J. Tobin, J. Elizalde, F. Palizas, C. M. David, J. Pimentel, M. González, L. Soto, G. D’Empaire, P. Pelosi, Incidence, risk factors and outcome of barotrauma in mechanically ventilated patients., Intensive care medicine 30 (4) (2004) $612-9$.

[77] M. Boussarsar, G. Thierry, S. Jaber, F. Roudot-Thoraval, F. Lemaire, L. Brochard, Relationship between ventilatory settings and barotrauma in the acute respiratory distress syndrome., Intensive care medicine 28 (4) (2002) 406-13.

[78] C. S. Poon, Optimality Principle in Respiratory Control, in: Proceedings of the 1983 American Control Conference., Vol. 1, IEEE, 1983, pp. 36-40.

[79] M. A. Mañanas, A. M. Hernández, R. Rabinovich, S. Benito, P. Caminal, Modeling and evaluation of respiratory and muscle pattern during hypercapnic stimulus, in: Annual International Conference of the IEEE Engineering in Medicine and Biology - Proceedings, Vol. 26 VI, 2004, pp. 3913-3916.

[80] C. Tin, G. Song, C.-S. Poon, Hypercapnia attenuates inspiratory amplitude and expiratory time responsiveness to hypoxia in vagotomized and vagal-intact rats, Respiratory Physiology \&amp; Neurobiology 181 (1) (2012) 79-87. 
[81] A. Albanese, N. W. Chbat, M. Ursino, Transient respiratory response to hypercapnia: Analysis via a cardiopulmonary simulation model, in: Engineering in Medicine and Biology Society, EMBC, 2011 Annual International Conference of the IEEE, IEEE, 2011, pp. 2395-2398.

[82] W. J. Reynolds, H. T. Milhorn, G. H. Holloman, Transient ventilatory response to graded hypercapnia in man, Journal of Applied Physiology 33 (1) (1972) 47-54.

[83] U. E. Ruttimann, W. S. Yamamoto, Respiratory airflow patterns that satisfy power and force criteria of optimality, Annals of biomedical engineering 1 (2) (1972) $146-159$.

[84] A. S. C. Patvardhan, Sulabh Bansal, Solution of "Hard" Knapsack Instances Using Quantum Inspired Evolutionary Algorithm, in: Research Methods: Concepts, Methodologies, Tools and Applications, 2015, p. 1075.

[85] E. J. M. Campbell, E. Agostoni, J. Newsom-Davis, The respiratory muscles mechanics and neural control. 2, Auflage, Lloyd-Luke LTD, London.

[86] R. L. Vender, M. F. Betancourt, E. B. Lehman, C. Harrell, D. Galvan, D. C. Frankenfield, Prediction equation to estimate dead space to tidal volume fraction correlates with mortality in critically ill patients., Journal of critical care 29 (2) (2014) 317.e1-3.

[87] M.-N. Fiamma, C. Straus, S. Thibault, M. Wysocki, P. Baconnier, T. Similowski, Effects of hypercapnia and hypocapnia on ventilatory variability and the chaotic dynamics of ventilatory flow in humans, American Journal of PhysiologyRegulatory, Integrative and Comparative Physiology 292 (5) (2007) R1985R1993.

[88] F. B. Tancredi, I. Lajoie, R. D. Hoge, Test-retest reliability of cerebral blood flow and blood oxygenation level-dependent responses to hypercapnia and hyperoxia using dual-echo pseudo-continuous arterial spin labeling and step changes in the fractional composition of inspired gases., Journal of magnetic resonance imaging : JMRI (2015) 1-14. 